Bond University

Research Repository

\title{
Efficacy of habit-based weight loss interventions: a systematic review and meta-analysis
}

Cleo, Gina; Beller, Elaine ; Glasziou, Paul ; Isenring, Elisabeth; Thomas, Rae

Published in:

Journal of Behavioral Medicine

DOI:

10.1007/s10865-019-00100-w

Licence:

Other

Link to output in Bond University research repository.

Recommended citation(APA):

Cleo, G., Beller, E., Glasziou, P., Isenring, E., \& Thomas, R. (2020). Efficacy of habit-based weight loss interventions: a systematic review and meta-analysis. Journal of Behavioral Medicine, 43(4), 519-532. https://doi.org/10.1007/s10865-019-00100-w

\footnotetext{
General rights

Copyright and moral rights for the publications made accessible in the public portal are retained by the authors and/or other copyright owners and it is a condition of accessing publications that users recognise and abide by the legal requirements associated with these rights.

For more information, or if you believe that this document breaches copyright, please contact the Bond University research repository coordinator.
} 


\title{
Efficacy of habit-based weight loss interventions: a systematic review and meta-analysis
}

\author{
Gina Cleo ${ }^{1}$; Elaine Beller ${ }^{1}$; Paul Glasziou ${ }^{1}$; Elisabeth Isenring ${ }^{2}$; Rae Thomas ${ }^{1}$ \\ 1. Institute for Evidence-Based Healthcare, Bond University, Gold Coast, Australia. \\ 2. Faculty of Health Sciences and Medicine, Bond University, Gold Coast, Australia.
}

\begin{abstract}
Purpose: Habit-based interventions are a novel and emerging strategy to help reduce excess weight in individuals with overweight or obesity. This systematic review and meta-analysis aims to determine the efficacy of habit-based interventions on weight loss.
\end{abstract}

Methods: We identified potential studies through electronic searches in February 2019. Included studies were randomized /quasi randomized controlled trials comparing weight loss interventions founded on habit-theory with a control (active or non-active) and enrolled adults with overweight or obesity (body mass index $\geq 25 \mathrm{~kg} / \mathrm{m}^{2}$ ).

Results: Five trials (630 participants) met our inclusion criteria. After the intervention period (range 8-14 weeks), weight loss was modest but statistically different between groups (1.4kg [95\% confidence interval $0.5,2.3 ; P=0.004]$ ) favoring habit-based interventions. Intervention groups averaged $2.5 \mathrm{~kg}$ weight loss (range 1.7 to $6.7 \mathrm{~kg}$ ) compared with control $1.5 \mathrm{~kg}$ (range 0.4 to $5.8 \mathrm{~kg}$ ) and were 2.4 times more likely to achieve clinically beneficial weight loss ( $\geq 5 \%$ weight reduction).

Conclusions: Average weight losses in adults with overweight and obesity using habit-based interventions appear to be of clinical benefit. There were statistically significant differences in weight loss between habit-based interventions and controls, post-intervention. Longer studies powered to examine at least 12-month follow-up are required to more accurately determine the role of habit-based interventions on long-term weight loss maintenance.

Key words: behavioral science; habits; obesity; overweight; systematic review; weight loss 
Cleo, 2019. Efficacy of habit-based weight loss interventions. Systematic Reviews

\section{Background}

Given the increasing prevalence of overweight and obesity, and the lack of consistent long-term weight loss success (Curioni \& Lourenceo, 2005; Montesi et al, 2016), the scope of research to explore novel strategies for effective intervention has broadened. A synthesis and comparison of these emergent weight management strategies is essential.

There is overwhelming evidence that in individuals with overweight and obesity, modest weight loss of $\geq 5 \%$ reduction in body weight is associated with significant health benefits, especially when maintained over time (Avenell et al, 2004; NHMRC, 2013). Recent studies have demonstrated the potential for habit-based interventions to positively influence health behaviors and reduce excess weight (Cleo et al, 2017; Lally et al, 2010; McGowan et al, 2013). Habits are automatic behaviors which have been triggered in response to a contextual cue (Kwasnicka et al, 2016). Habits are formed by repeatedly performing a behavior in a consistent context; this creates a mental association between the behavior and the context (Neal et al, 2006). The reinforcement of this context-behavior association creates an easily accessible response in our memory, such that alternative behaviors become less accessible (Gardner, 2014). Eventually, the mere perception of the context automatically triggers the responding behavior. Unlike lifestyle behaviors, which require deliberative and intentional thought, habitual behaviors are subconscious reactions that were initially instigated with intention but now are performed mindlessly (e.g., eating popcorn at the cinema or lunch at midday) (Rothman et al, 2009). Habit formation involves a gradual shift in cognitive control from intentional to automatic processes (Nilsen et al, 2012). Daily eating and exercise behaviors largely consist of mindless, automatic habits as are triggered by common cues such as time of day, location or preceding action (Van't Reit et al, 2011). These automatic behaviors occur without reflective decision making and increase with repetition of the behavior. Since habitual behavior is detached from motivational or volitional control (Gardner, 2016), habits are more likely to override intention in predicting behavior; the frequency of past behavior plays a significant role in predicting future behavior (Danner et al, 2008; Ji MF \& Wood, 2007). 
Cleo, 2019. Efficacy of habit-based weight loss interventions. Systematic Reviews

Habit-based interventions will generally include an 'initiation', 'learning' and 'stability' phase (Gardner et al, 2012). Habit formation begins with an 'initiation phase', during which the new chosen behaviour and the context in which that behaviour will be performed are selected; for example, 'eat a piece of fruit with breakfast', or 'go for a 30-minute walk at 7am'. Automaticity develops in the subsequent 'learning phase', during which the behaviour is repeated in the chosen context to strengthen the context-behaviour association (self-monitoring through a habit tracker is beneficial [Burke, 2011]). Finally, habit formation is accomplished in the 'stability phase', at which the behaviour persists over time with minimal conscious thought or effort (Gardner et al, 2012). Interventions that are founded on habit-change theory may therefore be important for weightmanagement as habitual behaviors are elicited automatically and are as a result, likely to be maintained (Duhigg, 2013; Lally et al, 2011).

This systematic review and meta-analysis is the first to assess the efficacy of habit-based weight loss interventions on weight loss outcomes. Our primary objective is to determine the efficacy of habitbased interventions on weight reduction in adults with overweight and obesity. Secondary outcomes are to determine: 1) the efficacy of habit-based interventions on weight loss maintenance in adults with overweight and obesity compared with control; 2) the proportion of participants achieving clinically beneficial weight loss and weight loss maintenance ( $\geq 5 \%$ total body weight loss) and; 3 ) subgroup analysis comparing habit-based interventions with active and non-active controls.

\section{Methods}

\section{Data sources and searches}

An experienced medical librarian conducted electronic searches within five databases, PubMed, Embase, CINAHL, Cochrane CENTRAL and Web of Science, in February 2019. Key search terms used were: habit (formation or disruption or based or breaking or break) and weight loss (or weight control or weight reduction). The systematic review protocol registration and search strategy are 
Cleo, 2019. Efficacy of habit-based weight loss interventions. Systematic Reviews

provided in the supplementary File 1 and File 2 respectively. A manual search through reference lists of included studies and other relevant articles was also performed. No language restrictions or publication dates were applied.

Studies included in this review were randomized or quasi-randomized. These studies used habit-based weight-loss interventions that focused specifically on habit-change as the conceptual theory; therefore, habit-change was the primary strategy for behavior change and subsequent weight loss. Habit-based interventions included explicitly forming new habits (through triggered repetition of the desired behavior), breaking old habits (through intentional routine/trigger disruption), or both. Randomized studies were eligible if they 1) enrolled participants with overweight or obesity defined as $\mathrm{BMI} \geq 25.0 \mathrm{~kg} / \mathrm{m}^{2} ; 2$ ) included participants over 18 years of age; 3 ) included a control group; and 4) reported quantified weight change outcomes at post-intervention.

\section{Study selection and data extraction}

All studies were screened independently against eligibility criteria by two reviewers. Screening of titles, abstracts and full-texts was conducted using EndNote X7.2.1. Articles that did not meet inclusion criteria were excluded. If uncertainty existed, the full text article was reviewed. The same two reviewers independently extracted and recorded data in pre-piloted data extraction forms. Disagreements were resolved by consensus and discussion. Extracted data included study design, setting, participant characteristics, intervention and comparator characteristics and weight change outcomes. If there were inadequate data for a given outcome, the corresponding author was contacted via e-mail at least twice and the data were requested. This manuscript followed the PRISMA 2009 Checklist (available in the Supplementary Material). 
Cleo, 2019. Efficacy of habit-based weight loss interventions. Systematic Reviews

\section{Quality assessment}

Risk of bias of included studies was independently assessed by two reviewers using the Cochrane Collaboration Risk of Bias tool (Higgins \& Green, 2011). Additionally, we uploaded included studies to RobotReviewer (www.robotreviewer.net/) to confirm accuracy of manual risk of bias assessment. To enhance the usability of this review, we extracted and reported summaries of the main intervention components for each study using the items from the Template for Intervention Description and Replication (TIDieR) checklist (Hoffmann et al, 2017).

\section{Data analyses}

Data were analyzed using available cases in Review Manager 5.3 (The Cochrane Collaboration, 2014). For analyses of weight change, between group mean differences were extracted or calculated per group, from baseline to post-intervention (primary outcome) and from baseline to last available follow-up (secondary outcome). The proportion of participants achieving $\geq 5 \%$ total body weight reduction (clinically important weight loss) was extracted from reported data and presented as a risk ratio comparing intervention with control. We conducted subgroup analyses to examine mean differences in weight change between the intervention groups and active compared with non-active controls. We also conducted post-hoc subgroup analyses comparing mean differences in interventions which used only forming habits as their theoretical basis compared with interventions that used forming habits in combination with breaking habits. If multiple follow-up assessments were reported, the last available follow-up (i.e., longest duration from baseline) was used.

\section{Effect size heterogeneity}

The $\mathrm{I}^{2}$ statistic was used to assess variability in effect size among studies (heterogeneity). We expected statistically significant heterogeneity due to methodological differences in the studies, including variations in study duration and intervention characteristics. A random effects model was therefore used to synthesize the data. There were too few studies to explore the impact of study 
Cleo, 2019. Efficacy of habit-based weight loss interventions. Systematic Reviews

variables on heterogeneity using meta-regression analysis; therefore, we described the differences in methods, duration and intervention characteristics in narrative and tabular form.

\section{Results}

The systematic search identified 730 potentially relevant studies (Figure 1). Of these, 229 were duplicates. Therefore, 501 titles and abstracts were screened for eligibility; 490 did not meet inclusion criteria. Of the 11 full text articles assessed, 2 were excluded as they were duplicates or preliminary findings of the included studies, 2 because the intervention used was not habit-based, and 2 because the study was not randomized (study details provided in supplementary file [See Supplementary Table 1 for descriptive data of excluded studies]). Five articles were included in the analyses.

\section{Study characteristics}

The studies reported results from Australia (Cleo et al, 2018), USA (Carels et al, 2011; Carels et al, 2014) and UK (Beeken et al, 2017; Lally \& Gardner, 2013). Most studies were conducted in university clinics (Carels et al, 2011; Carels et al, 2014; Cleo et al, 2018; Lally et al, 2008) and one study in a primary care setting (general practice clinics) (Beeken et al, 2017). The five studies included 630 participants (study size ranged from 43 to 383), with a pre-intervention mean body weight, ranging from 90 to $109 \mathrm{~kg}$ (BMI $31-38 \mathrm{~kg} / \mathrm{m}^{2}$ ), mean age ranged from 44 to 66 years and all studies reported a majority of female participants, $66-80 \%$. Table 1 displays the included studies and their characteristics.

\section{Interventions}

Studies based their interventions on forming new habits (Ten Tops Tips) (Beeken et al, 2017; Cleo et al, 2018; Lally et al, 2008), breaking old habits (Do Something Different) (Cleo et al, 2018), or a 
Cleo, 2019. Efficacy of habit-based weight loss interventions. Systematic Reviews

combination of both (Transforming Your Life) (Carels et al, 2011; Carels et al, 2014) (Table 1). One study (Cleo et al, 2018) compared two different interventions (one which formed new habits and the other broke old habits) with a control group. There were no significant differences in weight loss and weight loss maintenance between the two habit-based interventions groups. Therefore, we grouped the intervention results together for the primary and secondary outcome measures.

Ten Top Tips (TTT): based on habit-formation theory, TTT encouraged daily repetition of ten behaviors, proposed to create a negative energy balance and subsequent weight loss. The behaviors (tips), included: keep to a meal routine; eat reduced fat foods; walk 10,000 steps a day; pack a healthy snack; check food labels; watch portion sizes; stand up for 10 minutes in every hour; choose low calorie drinks; be mindful when eating, and; eat 5 portions of fruit and vegetables a day. To encourage habit development, participants were advised to plan ahead to effectively incorporate the tips into their daily routines and repeat the behaviors in a consistent context. Participants were encouraged to repeat as many of the tips as they could, every day for the intervention period (8-12 weeks).

Do Something Different (DSD): focused on increasing participants' behavioral flexibility by breaking daily habits purported to play a role in unhealthy dietary and exercise behaviors. Participants were sent an unpredictable task to perform, via text message and/or email, three to four times a week. The tasks required them to do something different to expand their behavioral repertoire and were not diet or exercise related. Examples include: 'drive a different way to work today', 'choose a charity or local group to help' or 'write a short story on any subject'.

Transforming Your Life (TYL): used environmental modification to promote the formation of new, healthy habits (through development of predictable and sustainable weight-loss related routines), as well as disruption of old, unhealthy habits (by changing established routines that support unhealthy habits). Individuals were taught to create their personal food and exercise environment in a manner that minimizes unhealthy eating and sedentary behavior cues, maximizes healthy eating and exerciserelated cues, and encourages automatic responding to goal-related cues. Each week, selected 
Cleo, 2019. Efficacy of habit-based weight loss interventions. Systematic Reviews

environmental factors that have been empirically recognized to influence eating (e.g. visibility, variety, serving utensils, abundance, and convenience) were systematically targeted for modification.

Intervention duration ranged from 8 to 14 weeks and follow-up 6 to 24 months. Control groups in the included studies were either, active (non-habit-based weight loss programs [Carels et al, 2011; Carels et al, 2014] and lifestyle advice with or without community referral [Beeken et al, 2017]) or nonactive (wait list [Cleo et al, 2018; Lally et al, 2008]).

Weight change

Primary outcome. Participants who completed a habit-based intervention, ranging between 8 and 14 weeks, weighed on average 1.4kg lighter than the control participants at post-intervention (Figure 2). Intervention participants achieved an overall average weight loss of $2.5 \mathrm{~kg}$ (range 1.7 to $6.7 \mathrm{~kg}$ ) compared with the $1.5 \mathrm{~kg}$ mean loss of control participants (range 0.4 to $5.8 \mathrm{~kg}$ ). Weight loss was significantly different between intervention and control groups (mean difference $-1.4 \mathrm{~kg}$ [95\% CI -2.3, $-0.5 ; P=0.004])$.

As expected, there was a statistically significant heterogeneity among studies post-intervention $\left(\mathrm{I}^{2}=\right.$ $71 \%$ ). Statistical exploration of heterogeneity was unable to be conducted due to a small number of included studies. However, as a sensitivity analysis, we removed Cleo et al, 2018 and Lally et al, 2008 from the forest plot as they were the only two studies which used wait-list control groups instead of active-controls. When we removed these two studies, the $\mathrm{I}^{2}$ was $0 \%$. We therefore suspect heterogeneity between studies was due to the difference in the use of active and non-active control groups.

Secondary outcomes. Three of the five studies measured weight loss maintenance in both the intervention and control groups at follow-up and were included in a meta-analysis (Beeken et al, 2017; Carels et al, 2011; Carels et al, 2014). All control groups included in the meta-analysis were active. After a 6-24-month follow-up, weight loss maintenance in the intervention group was $3.4 \mathrm{~kg}$ 
(range 2.2 to $8.9 \mathrm{~kg}$ ) and $3.1 \mathrm{~kg}$ (range 3.0 to $4.2 \mathrm{~kg}$ ) in active-controls (See Supplementary Figure 1 for comparison of weight loss at follow-up between intervention and control groups). However, weight loss maintenance was not statistically significant between the intervention and active-control groups (mean difference $0.1 \mathrm{~kg}[95 \% \mathrm{CI}-2.5,2.4 ; P=0.96]$ ).

The two studies (Cleo and Lally) where only intervention group data were reported at follow-up (and not control group data) achieved mean weight loss maintenance of $5.1 \mathrm{~kg}$ and $3.8 \mathrm{~kg}$ after 12 and 8 months follow-up, respectively.

Three of the five studies reported on the proportion of participants achieving clinically beneficial weight loss ( $\geq 5 \%$ total body weight reduction); two of the three at post-intervention (Beeken et al, 2017; Cleo et al, 2018) and all three at follow-up (Beeken et al, 2017; Cleo et al, 2018; Lally et al, 2008) (Supplementary Figure 2). At post-intervention, 16-35\% of participants in the intervention group and $4-8 \%$ in the control group had achieved clinically beneficial weight loss. Participants in the intervention group were therefore, 2.4 times (risk ratio) more likely to achieve clinically beneficial weight loss than control participants (Figure 3). At follow up, 27-65\% of participants in the intervention group and $26 \%$ in an active-control group had achieved and maintained a reduction of $\geq 5 \%$ total body weight (Supplementary Figure 2).

We observed a moderate heterogeneity amongst studies in the follow-up analysis, however this was not statistically significant $\left(\mathrm{I}^{2}=50 \%\right)$.

\section{Subgroup analysis}

Analyses were completed for two subgroups: active vs non-active control groups and forming habits vs forming + breaking habits. There was a $0.8 \mathrm{~kg}$ mean difference favoring the intervention group, when comparing habit-based interventions with active controls $(P=0.01)$. The mean difference increased to $2.1 \mathrm{~kg}$ when comparing habit-based interventions with non-active controls $(P=<0.001)$ (Figure 4). 
Cleo, 2019. Efficacy of habit-based weight loss interventions. Systematic Reviews

Interventions which used forming new habits as the theoretical basis of their intervention (Beeken et al, 2017; Cleo et al, 2018; Lally et al, 2008), showed a statistically significant mean difference when compared with controls (mean difference $1.4 \mathrm{~kg} ; P=0.001$ ), however two of three of these used waitlist controls. Interventions which used forming new habits in combination with breaking old habits did not show a statistically or clinically meaningful difference compared with controls (mean difference 1.2kg; $P=0.37$ ) (Carels et al, 2011; Carels et al, 2014; Cleo et al, 2018) (See Supplementary Figure 3 for subgroup analysis).

\section{Quality of included studies}

Four studies were randomized controlled trials (Beeken et al, 2017; Carels, 2011; Carels, 2014; Cleo et al, 2018; Lally et al, 2008) and one was randomized according to intervention commencement date (Lally et al, 2008) (Table 1). As expected, participants and investigators of all studies were aware of the intervention they were receiving or delivering resulting in all studies rating as 'high risk' of bias for blinding of participants and study personnel. We displayed risk of bias outcomes for random sequence generation, allocation concealment, blinding of participants and personnel, blinding of outcome assessment, incomplete outcome data and selective reporting (Figure 5). A TIDieR intervention summary was completed for each of the 5 studies included in this review (Table 2). This summary describes why the intervention was initiated, what methods and materials were used, and details of intervention fidelity, amongst other intervention information.

\section{Discussion}

Despite our intentions, habits influence a large proportion of our daily behaviors. If we were able to change our eating habits - where, when and why we eat, - we might be able to manage our weight more effectively. Therefore, determining if habit-change interventions are effective for weight loss and weight loss maintenance is important. We systematically evaluated the efficacy of habit-based 
Cleo, 2019. Efficacy of habit-based weight loss interventions. Systematic Reviews

interventions for weight loss and weight loss maintenance in individuals with overweight and obesity. There was a statistically significant difference in weight loss between intervention and control participants after an 8 to 14 week intervention period. Participants in the intervention group were 2.4 times more likely to achieve clinically beneficial weight loss than the control group.

The long-term effects of habit-based interventions on weight loss maintenance were difficult to conclude as the interventions were compared with active-control groups (non-habit-based weight loss programs (Carels et al, 2014; Lally et al, 2008) and lifestyle advice with or without community referral (Beeken et al, 2017)). The two studies which used non-active controls (wait-list) were not included in the follow-up analysis as they did not measure or analyze these data. However, the singlearmed results from these two studies show that the participants had in fact achieved weight-loss maintenance (Cleo et al, 2018; Lally et al, 2008).

In the current analyses, forming habits achieved greater weight loss when compared with forming+breaking habit interventions. However, both studies of forming+breaking habits (Carels et al, 2014; Carels et al, 2011) were compared with active controls. Therefore, studies comparing forming+breaking habits compared with non-active controls are required to determine robust measures of outcomes.

Our study is strengthened by our procedures. We searched five databases and contacted authors for missing data or unclear practices. We also assessed the quality of the studies using the Cochrane Risk of Bias tool (Higgins \& Green, 2011). Overall, the risk of bias was well-reported in the studies; however, there were some inconsistencies. One study reported the outcome assessor was not blind to group allocation when conducting post-assessments and two studies did not report on this (Carels et al, 2011; Carels et al, 2014) and were rated as unclear risk for detection bias. Due to the nature of the interventions, it is not possible to blind participants and personnel to treatment allocations, therefore all studies were rated as high risk of bias for blinding. However, clinic-measured weight is objective rather than self-reported so the magnitude of bias associated with inadequate blinding of participants is not likely to affect outcomes (Higgins \& Green, 2011). Finally, knowing an intervention is effective 
is inconsequential if it is not described well for clinicians to use. A key strength of this review was including a TIDieR summary of interventions. This summary provided a description of the main intervention components for each of the included studies for clinicians to make important judgements about their ability to implement these interventions in their place of work.

The published literature on habit-based weight loss interventions is scant. This systematic review was limited by the small number of published studies, using habit-based interventions for weight management in individuals with overweight and obesity. Interventions which focus on habit-change theory are a novel and emerging approach to weight management. We anticipate seeing more included studies in future updates of this review. Also, there were varied levels of heterogeneity $\left(\mathrm{I}^{2}=0 \%\right.$ to $82 \%$ ['might not be important' to 'considerable heterogeneity']) (Higgins \& Green, 2011) in the included studies. There is considerable conceptual heterogeneity among the included studies, with different behavior change techniques required for forming new habits compared with disrupting old habits (Lally \& Gardner, 2013). Despite these differences, we meta-analyzed the data as the novelty in all the included studies is that they are founded on habit-change theory (whether forming new habits or breaking old ones). Due to the limited number of studies, meta-regression could not be conducted to explore study variability that might have contributed to the heterogeneity.

The weight loss-weight regain cycle is common. Previous systematic reviews show that after a lifestyle program, commonly diet and exercise, an average of $46-50 \%$ of weight loss is regained just 12-months post-treatment (Anderson et al, 2001; Barte et al, 2010; Curioni \& Lourenco, 2005) and much of the rest over the subsequent 3 years (Roque et al, 2013). However, unlike diet and exercise programs, interventions which are founded on habit-change theory encourage behavior to become 'second nature'; therefore, the new, healthy behaviors are more resistant to change.

Although more research is required to accurately determine the efficacy of habit-based interventions when compared with non-active control groups long-term, habit-change theory proposes that the addition of habit-change techniques to current clinical practice may increase the chances of long-term weight loss maintenance (Duhigg, 2013; Lally et al, 2011). For example, including contextual cues 
Cleo, 2019. Efficacy of habit-based weight loss interventions. Systematic Reviews

when goal setting. A contextual cue is used as a trigger for the subsequent habitual behavior and must be personalized to each individual's lifestyle and daily routines, e.g., "when I am eating, I will put away any distractions", or "at 7am, I will go for a 30-minute brisk walk". Using current clinical practice guidelines, with the addition of contextual cues, encourages an automatic response to those cues. Therefore, an individual is exposed to daily triggers which prompt them to perform the new desired behavior without depending on their memory or motivation.

\section{Conclusions}

Habit-based weight loss programs are more effective at achieving clinically beneficial weight loss than lifestyle advice, non-habit-based programs and waitlist post-intervention. The results presented in this systematic review are novel and provide a unique perspective from which to derive a new approach to weight management. Longer and more methodologically rigorous studies that are powered to examine at least a 12-month follow-up are required to determine the role of habit formation and disruption on weight loss maintenance. 
Cleo, 2019. Efficacy of habit-based weight loss interventions. Systematic Reviews

Trial registration: Prospero ID: CRD42017065589 Available from

https://www.crd.york.ac.uk/PROSPERO/display_record.asp?ID=CRD42017065589

Financial disclosure: GC was supported by an Australian Government Research Training Program Scholarship. RT and EB are supported by a NHMRC grant (APP1104136, APP1044904). PG is supported by a NHMRC Australian Fellowship grant (GNT1080042). LI received no funding with respect to this study.

Conflict of interests: The authors declare no conflict of interests.

Acknowledgements: We thank Ms Rebecca Simms for her invaluable assistance in data extraction and analysis. We also thank Mr Justin Clark for conducting our search.

\section{Supplementary Material}

PRISMA Checklist

Supplementary File 1: PROSPERO International prospective register of systematic reviews

Supplementary File 2: Search Strategy

Supplementary Table 1: Descriptive data of excluded studies

Supplementary Figure 1: Comparing weight loss $(\mathrm{kg})$ at follow-up between intervention and control groups

Supplementary Figure 2: Proportion of participants achieving $\geq 5 \%$ total body weight reduction at post-intervention and follow-up

Supplementary Figure 3: Subgroup analysis comparing weight loss $(\mathrm{kg})$ between forming habits and a combination of forming and breaking habits 
Cleo, 2019. Efficacy of habit-based weight loss interventions. Systematic Reviews

\section{References}

1. Anderson J.W., \& Konz E.C., Frederich R.C., Wood C.L. (2001). Long-term weight-loss maintenance: a meta-analysis of US studies. American Journal of Clinical Nutrition 74, 579584.

2. Avenell A., Brown T.J., McGee M.A., et al. (2004). What are the long-term benefits of weight reducing diets in adults? A systematic review of randomized controlled trials. Journal of Human Nutrition and Dietetics 17, 317-335.

3. Barte JC, ter Bogt NC, Bogers RP, et al. Maintenance of weight loss after lifestyle interventions for overweight and obesity, a systematic review. Obes Rev 2010; 11: 899-906.

4. Beeken RJ, Leurent B, Vickerstaff $\mathrm{V}$, et al. A brief intervention for weight control based on habit-formation theory delivered through primary care: results from a randomised controlled trial. Int J Obes 2017; 41: 246-254.

5. Burke LE, Wang J, Sevick MA. Self-Monitoring in Weight Loss: A Systematic Review of the Literature. J Am Diet Assoc. 2011;111(1):92-102.

6. Carels RA, Burmeister J M, Koball AM, et al. A randomized trial comparing two approaches to weight loss: Differences in weight loss maintenance. J Health Psychol 2014; 19: 296-311.

7. Carels RA, Young KM, Koball A, et al. Transforming your life: an environmental modification approach to weight loss. J Health Psychol 2011; 16: 430-438.

8. Cleo G, Hersch J, Thomas R. Participant experiences of two successful habit-based weightloss interventions in Australia: a qualitative study. BMJ Open 2018; 8.

9. Cleo G, Isenring L, Glasziou P, Beller E, Thomas R. Habit-based interventions for weight loss maintenance in adults with overweight and obesity: A randomized controlled trial. Int J Obes 2018.

10. Cleo G, Isenring L, Thomas R, Glasziou P. Could habits hold the key to weight loss maintenance? A narrative review. J Hu Nutr Diet 2017; 30: 655-664 
Cleo, 2019. Efficacy of habit-based weight loss interventions. Systematic Reviews

11. Curioni CC, Lourenco PM. Long-term weight loss after diet and exercise: a systematic review. Int J Obes 2005; 29: 1168-1174.

12. Danner UN, Aarts $\mathrm{H}$, de Vries NK. Habit vs. intention in the prediction of future behaviour: the role of frequency, context stability and mental accessibility of past behaviour. Br J Soc Psychol 2008; 47: 245-265.

13. Duhigg C. The power of habit: why we do what we do, and how to change. Cornerstone: London, 2013.

14. Gardner B. A review and analysis of the use of 'habit' in understanding, predicting and influencing health-related behaviour. Health Psychol Rev. 2014; 9: 277-295.

15. Gardner B. Habit Formation and Behaviour Change. Oxford Research Encyclopedia of Psychology. Oxford University Press, USA 2016.

16. Gardner B, Lally P, Wardle J. Making health habitual: the psychology of 'habit formation' and general practice. Br J Gen Pract. 2012; 62: 664-666.

17. Higgins J, Green S. (2011). Cochrane handbook for systematic reviews of interventions Version 5.1.0. URL http://handbook-5-1.cochrane.org/

18. Hoffmann TC, Oxman AD, loannidis JP, et al. Enhancing the usability of systematic reviews by improving the consideration and description of interventions. BMJ 2017; 358.

19. Ji MF, Wood W. Purchase and Consumption Habits: Not Necessarily What You Intend. J Consum Psychol 2007; 17: 261-276.

20. Kwasnicka D, Dombrowksi S, White M, Sniehotta F. Theoretical explanations for maintenance of behaviour change: a systematic review of behaviour theories. Health Psychol Rev 2016; 6: 277-296.

21. Lally P, Chipperfield A, Wardle J. Healthy habits: efficacy of simple advice on weight control based on a habit-formation model. Int J Obes 2008; 32: 700-707. 
Cleo, 2019. Efficacy of habit-based weight loss interventions. Systematic Reviews

22. Lally P, Van Jaarsveld CM, Potts HW, Wardle J. How are habits formed: Modelling habit formation in the real world. Eur J Soc Phsychol 2010; 40: 998-1009.

23. Lally P, Wardle J, Gardner B. Experiences of habit formation: A qualitative study. Psychol Health Med 2011; 16: 484-489.

24. Lally, P, Gardner B. Promoting habit formation. Health Psychology Review 2013; 7(SUPPL1): S137-S158.

25. McGowan L, Cooke LJ, Gardner B, Beeken RJ, Croker H, Wardle J. Healthy feeding habits: efficacy results from a cluster-randomized, controlled exploratory trial of a novel, habitbased intervention with parents. Am J Clin Nutr 2013; 98.

26. Montesi L, Ghoch M, Brodosi L, Calugi S, Marchesini G, Dalle Grave R. Long-term weight loss maintenance for obesity: a multidisciplinary approach. Diab Metab Syndr Obes. 2016; 9: $37-$ 46.

27. National Health and Medical Research Council. Clinical practice guidelines for the management of overweight and obesity in adults, adolescents and children in Australia, Australian Department of Health: Canberra Australia 2013.

28. Neal DT, Wood W, Quinn JM. Habits - A repeat performance. Curr Dir Psychol Sci. 2006; 15 : 198-202.

29. Nilsen P, Roback K, Brostrom A, Ellstrom PE. Creatures of habit: accounting for the role of habit in implementation research on clinical behaviour change. Implement Sci. 2012; 7: 53.

30. Review Manager (RevMan) [computer program]. The Cochrane Collaboration: Copenhagen 2014.

31. Roqué i Figuls M, Martinex Gracia L, Martinez-Zapata MJ, Pacheco R, Mauricio D, Bonfill CX. Interventions for treating overweight or obesity in adults: an overview of systematic review (Protocol). Cochrane Database Syst Rev 2013. 
Cleo, 2019. Efficacy of habit-based weight loss interventions. Systematic Reviews

32. Rothman, A. J., Sheeran, P., \& Wood, W. (2009). Reflective and automatic processes in the initiation and maintenance of dietary change. Annals of Behavioral Medicine 38 Suppl 1(1), S4-17.

33. Van't Reit, J., Sijtsema, S.J., Dagevos, H., \& De Bruijn, G. J. (2011). The importance of habits in eating behaviour. An overview and recommendations for future research. Appetite 57(3), 585-596. 
Cleo, 2019. Efficacy of habit-based weight loss interventions. Systematic Reviews

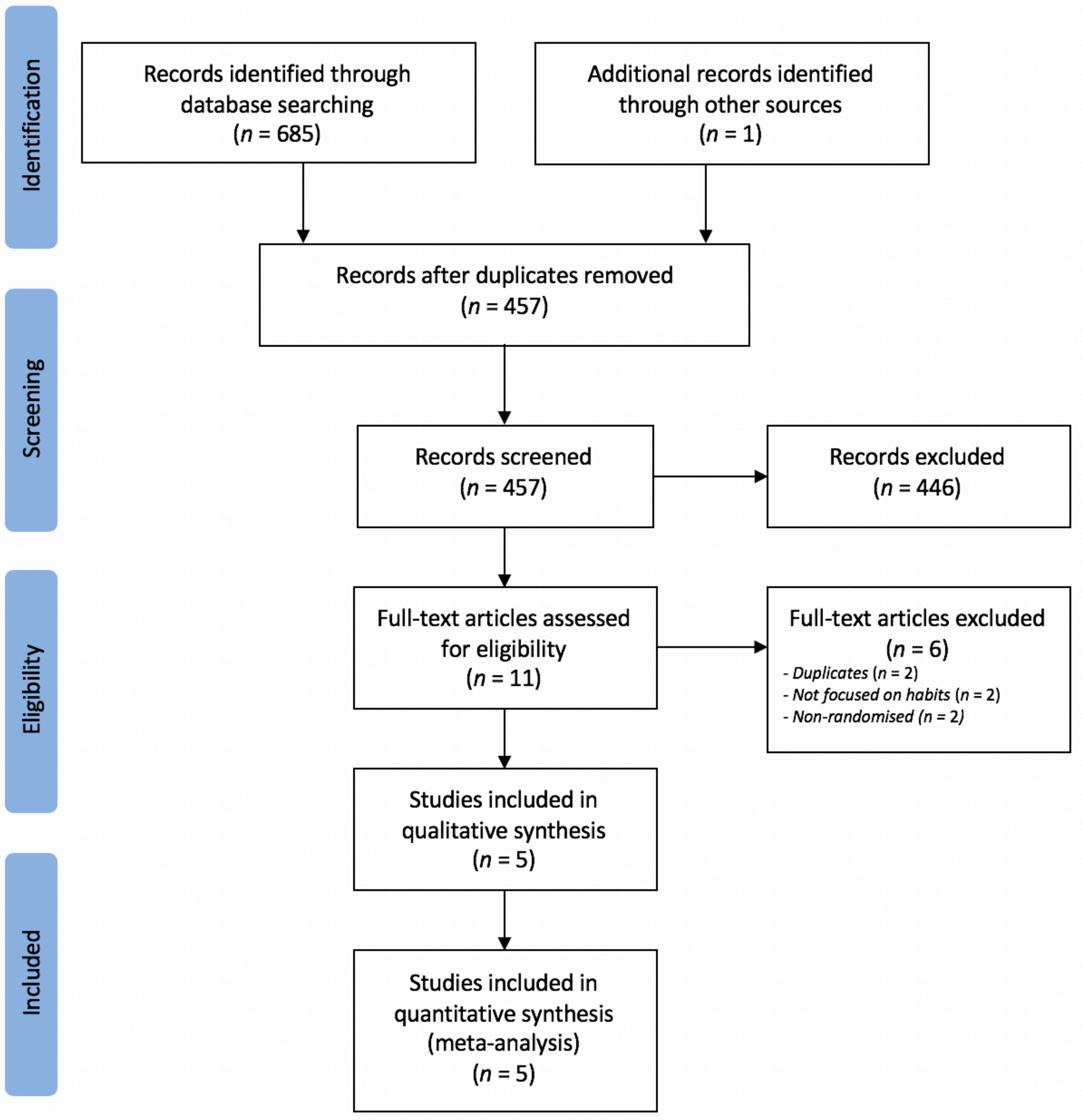

Figure 1 PRISMA Flow Diagram 
Cleo, 2019. Efficacy of habit-based weight loss interventions. Systematic Reviews

Figure 2 Comparing weight loss $(\mathrm{kg})$ at post-intervention between habit-based intervention and control groups

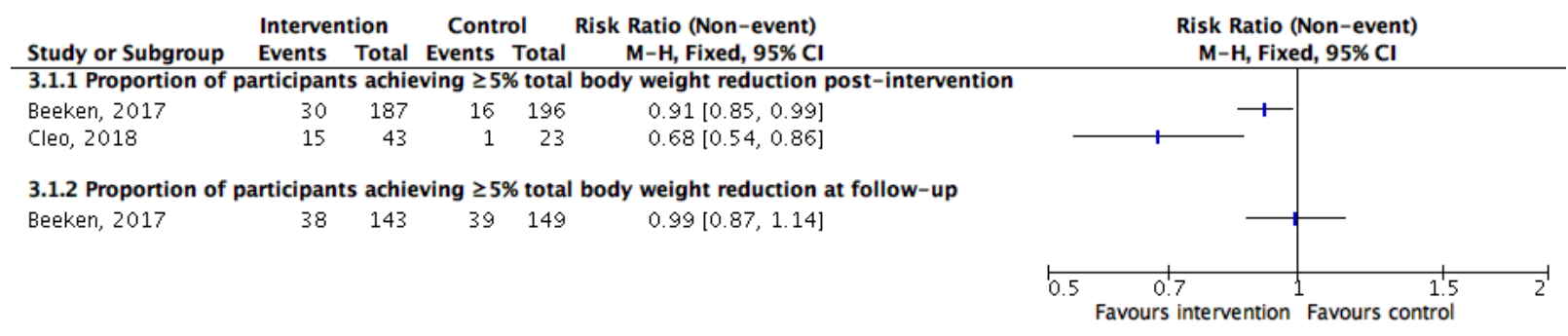

Figure 3 Subgroup analysis comparing effect size of the proportion of participants achieving $\geq 5 \%$ total body weight reduction at post-intervention and at follow-up

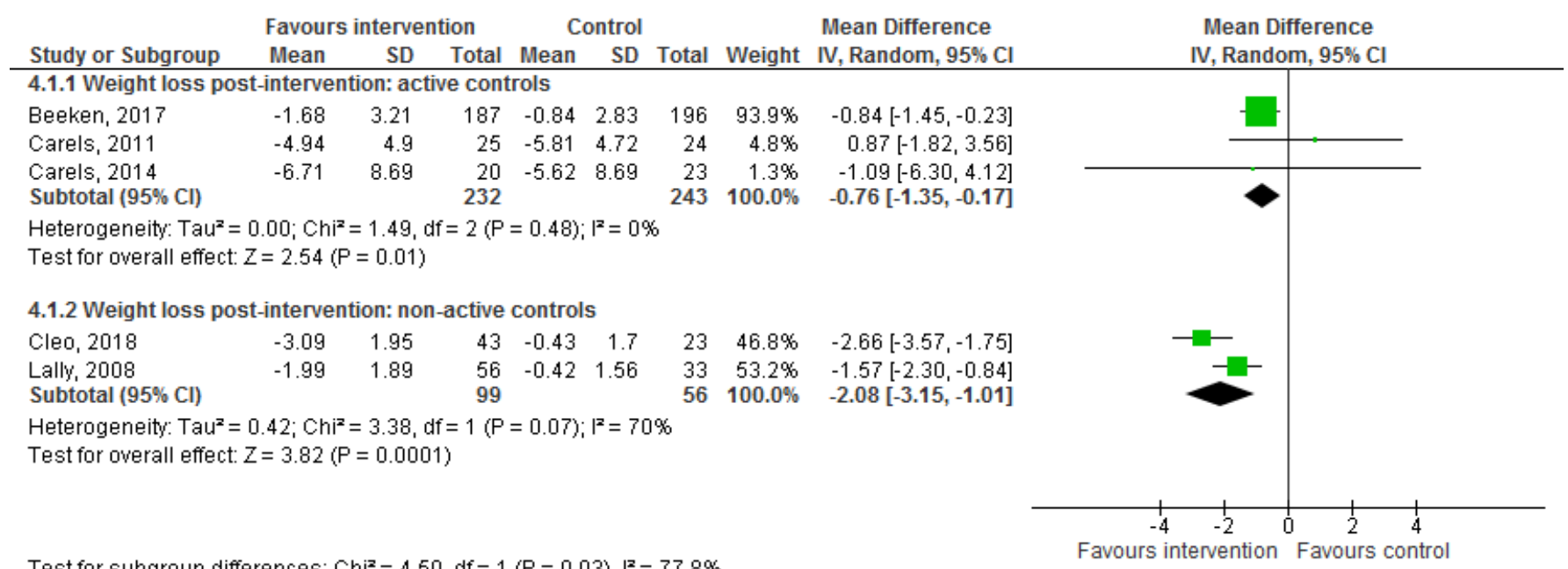

Test for subqroup differences: $\mathrm{Chi}^{2}=4.50, \mathrm{df}=1(\mathrm{P}=0.03), \mathrm{I}^{2}=77.8 \%$

Favours intervention Favours control

Figure 4 Subgroup analysis comparing weight loss $(\mathrm{kg})$ post-intervention between habit-based intervention and active vs non-active control groups

\begin{tabular}{llrrrrrrr} 
& \multicolumn{4}{c}{ Favours intervention } & \multicolumn{3}{c}{ Control } & \multicolumn{2}{c}{ Mean Difference } \\
Study or Subgroup & Mean & \multicolumn{1}{c}{ SD } & Total & Mean & SD & Total & Weight & IV, Random, 95\% Cl \\
\hline 4.1 .1 Weight loss post-intervention: active controls & & & & \\
Beeken, 2017 & -1.68 & 3.21 & 187 & -0.84 & 2.83 & 196 & $93.9 \%$ & $-0.84[-1.45,-0.23]$ \\
Carels, 2011 & -4.94 & 4.9 & 25 & -5.81 & 4.72 & 24 & $4.8 \%$ & $0.87[-1.82,3.56]$ \\
Carels, 2014 & -6.71 & 8.69 & 20 & -5.62 & 8.69 & 23 & $1.3 \%$ & $-1.09[-6.30,4.12]$ \\
Subtotal (95\% Cl) & & & 232 & & & 243 & $100.0 \%$ & $-0.76[-1.35,-0.17]$
\end{tabular}

Heterogeneity: Tau $^{2}=0.00 ; \mathrm{Chi}^{2}=1.49, \mathrm{df}=2(\mathrm{P}=0.48) ; \mathrm{I}^{2}=0 \%$

Test for overall effect: $Z=2.54(P=0.01)$

4.1.2 Weight loss post-intervention: non-active controls

$\begin{array}{lrrrrrrrr}\text { Cleo, } 2018 & -3.09 & 1.95 & 43 & -0.43 & 1.7 & 23 & 46.8 \% & -2.66[-3.57,-1.75] \\ \text { Lally, 2008 } & -1.99 & 1.89 & 56 & -0.42 & 1.56 & 33 & 53.2 \% & -1.57[-2.30,-0.84] \\ \text { Subtotal (95\% Cl) } & & & 99 & & & 56 & \mathbf{1 0 0 . 0 \%} & -2.08[-3.15,-1.01]\end{array}$

Heterogeneity: $\operatorname{Tau}^{2}=0.42 ; \mathrm{Chi}^{2}=3.38, \mathrm{df}=1(\mathrm{P}=0.07) ; \mathrm{I}^{2}=70 \%$

Test for overall effect: $Z=3.82(P=0.0001)$

Test for subqroup differences: $\mathrm{Chi}^{2}=4.50, \mathrm{df}=1(\mathrm{P}=0.03), \mathrm{I}^{2}=77.8 \%$

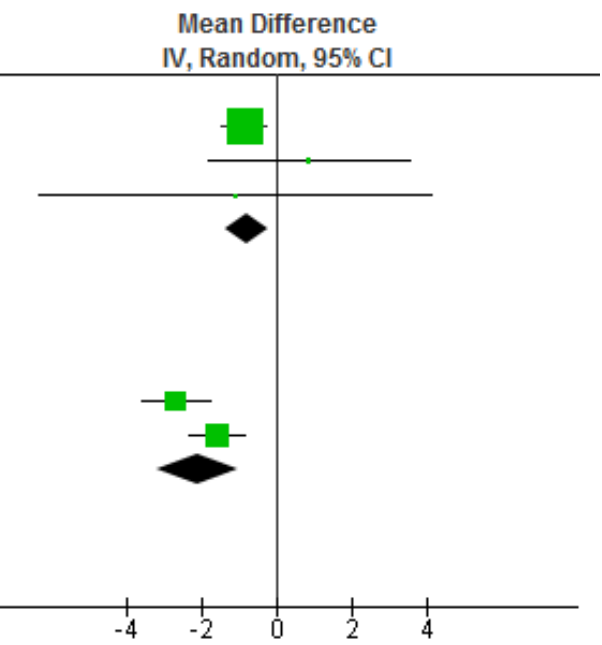

Favours intervention Favours control 
Cleo, 2019. Efficacy of habit-based weight loss interventions. Systematic Reviews

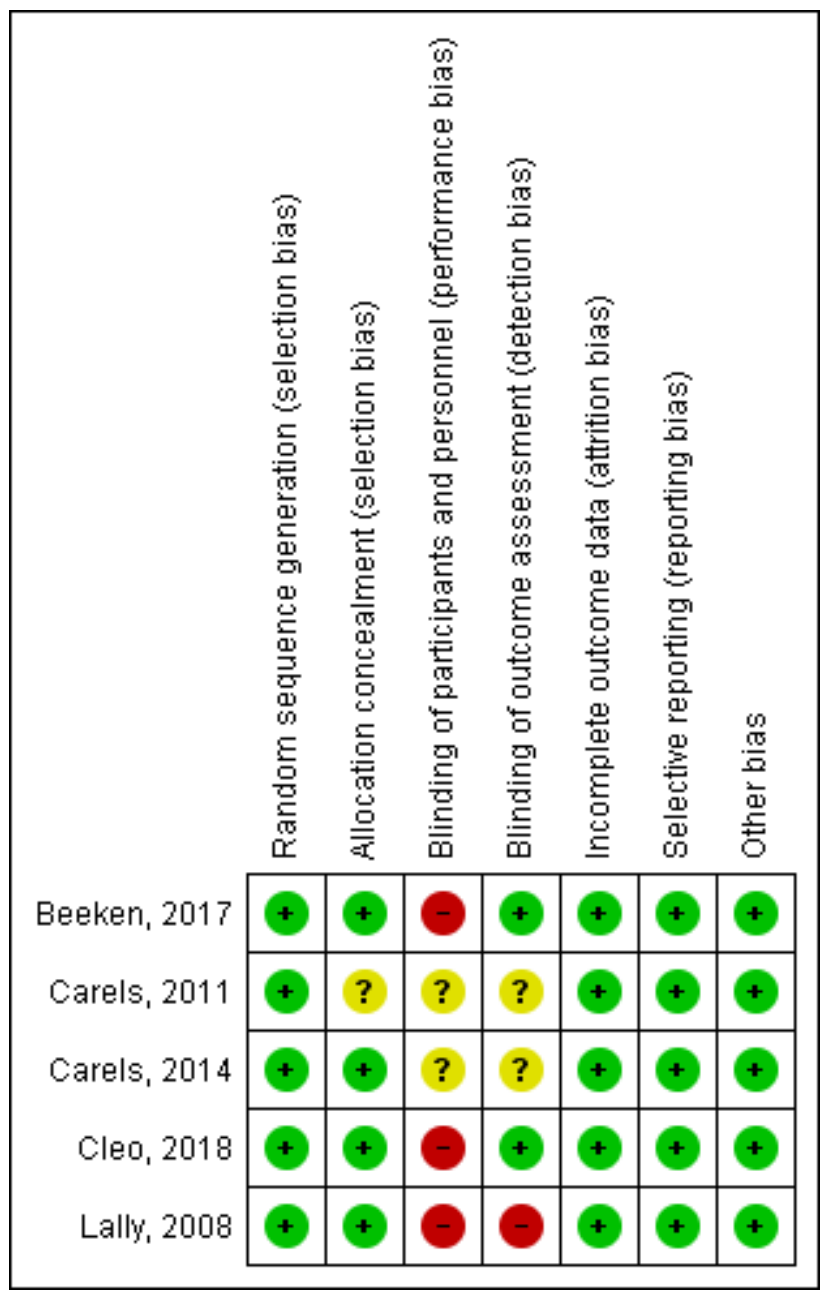

Figure 5 Risk of bias summary 


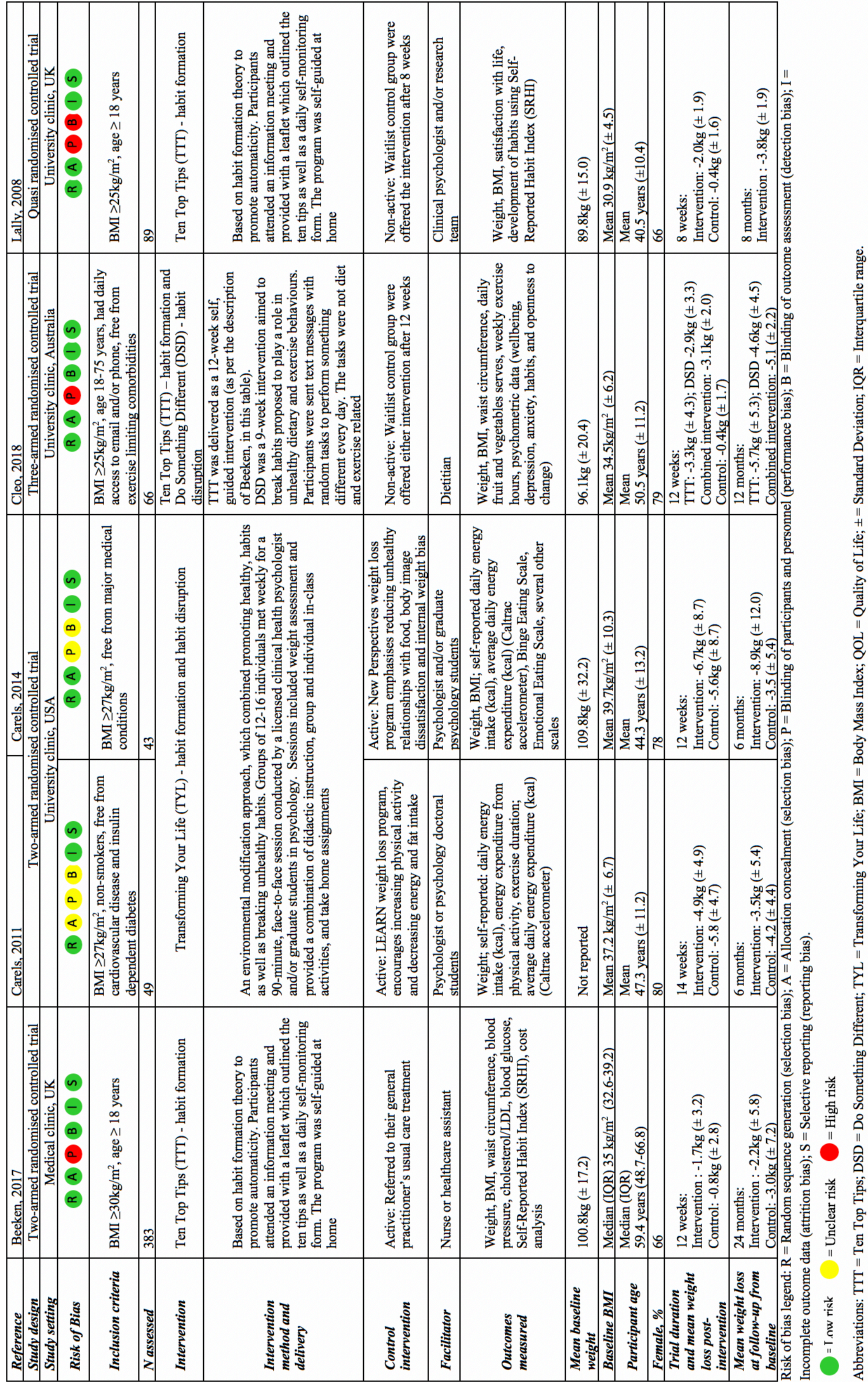

This is a post-peer-review, pre-copyedit version of an article published in Journal of Behavioral Medicine

The final authenticated version is available online at: http://dx.doi.org/10.1007/s10865-019-00100-w 


\begin{tabular}{|c|c|c|c|}
\hline 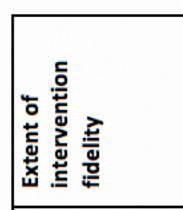 & 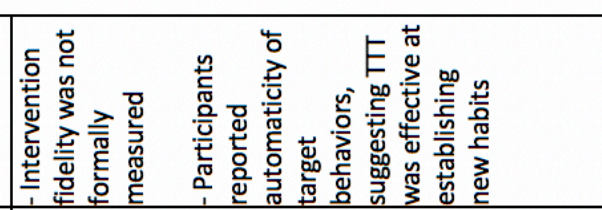 & 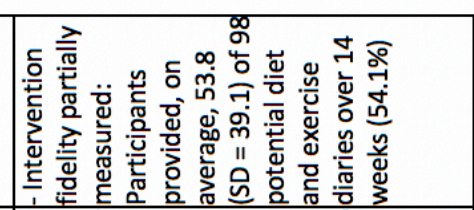 & 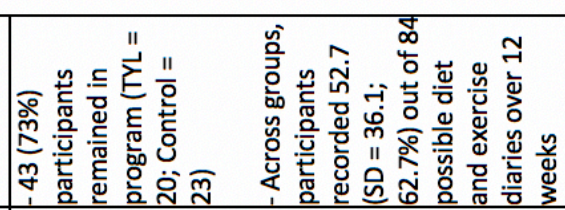 \\
\hline 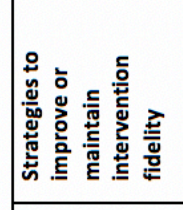 & 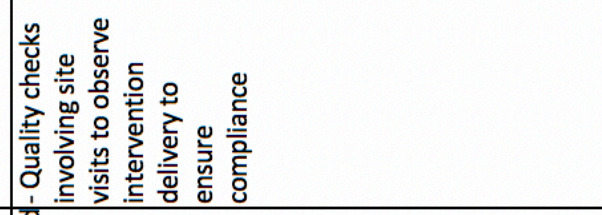 & 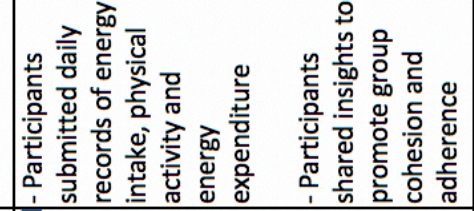 & \\
\hline 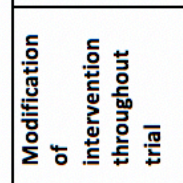 & 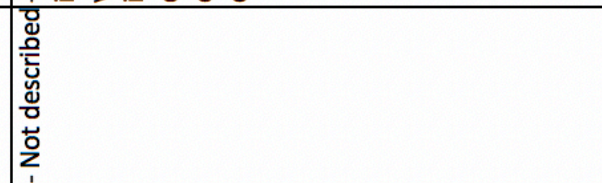 & & 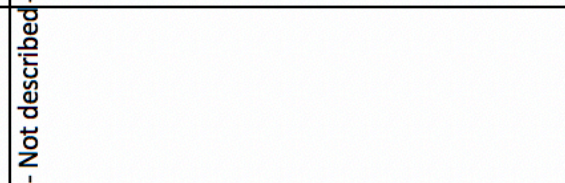 \\
\hline & 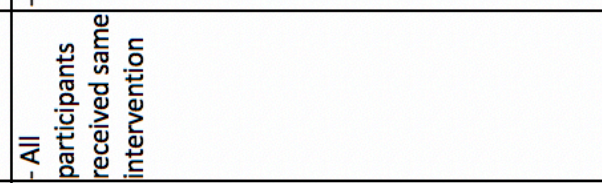 & 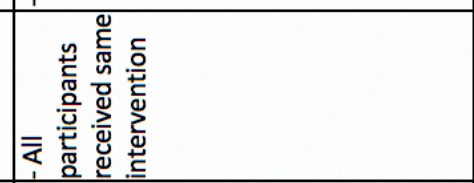 & 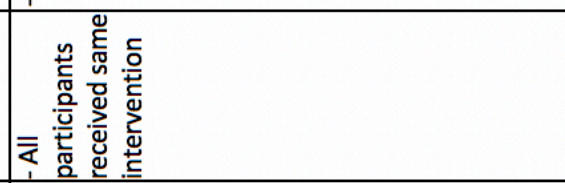 \\
\hline 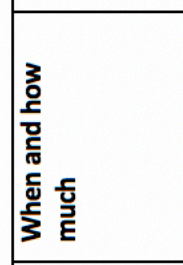 & 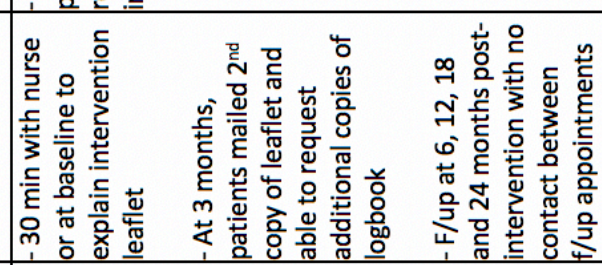 & 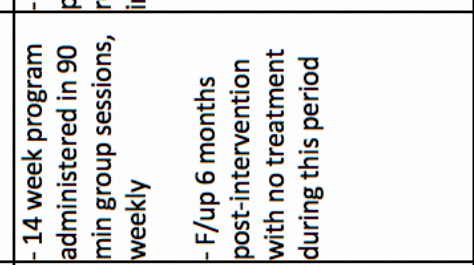 & 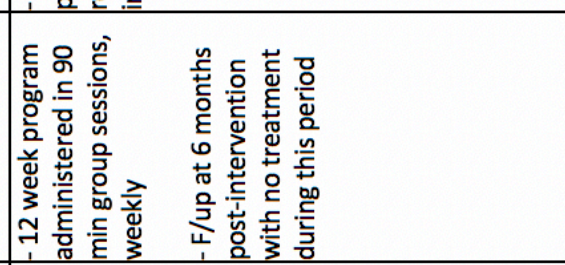 \\
\hline & 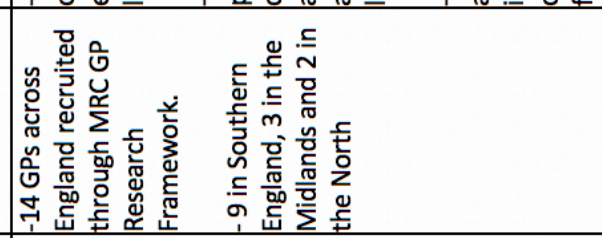 & 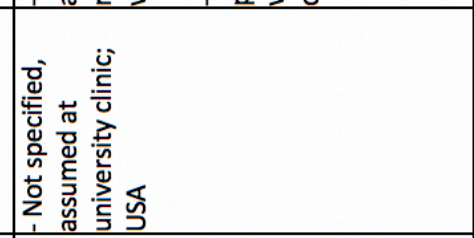 & 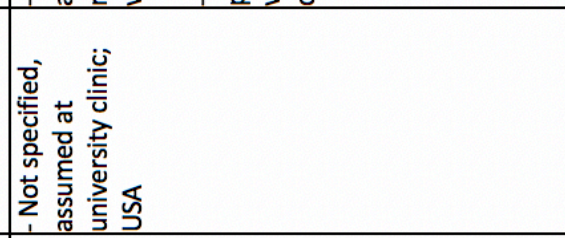 \\
\hline 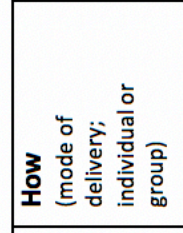 & 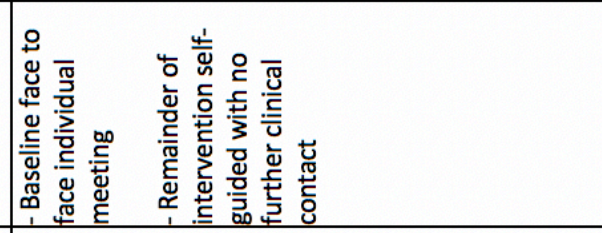 & 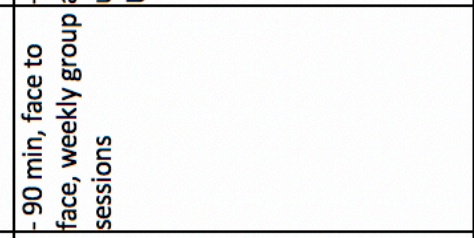 & 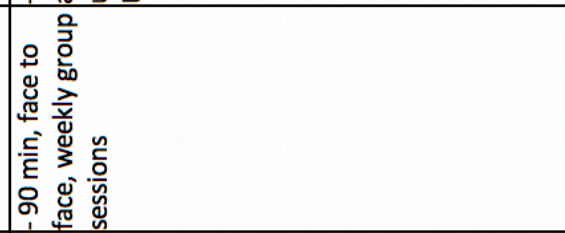 \\
\hline 这 & 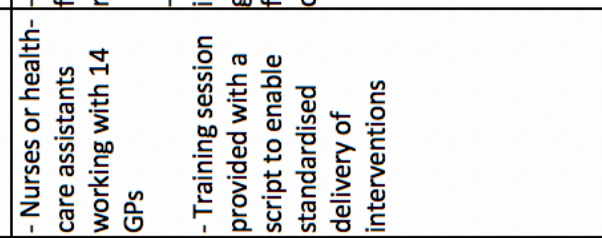 & 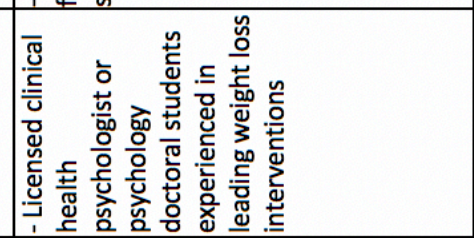 & 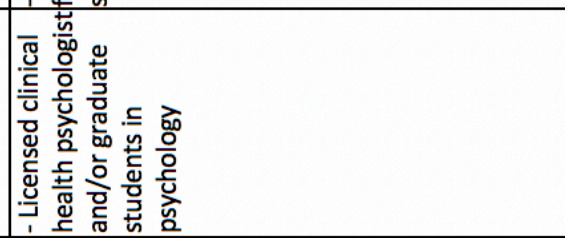 \\
\hline 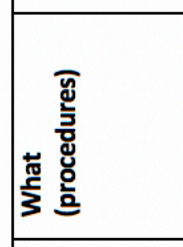 & 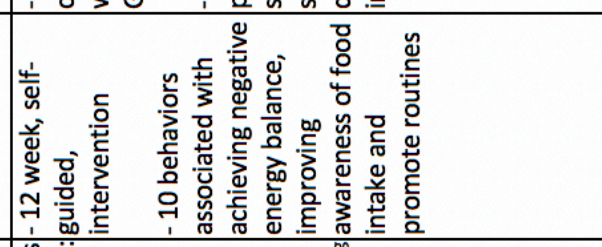 & 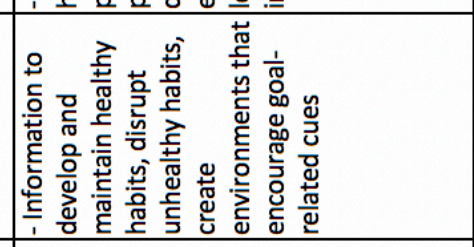 & 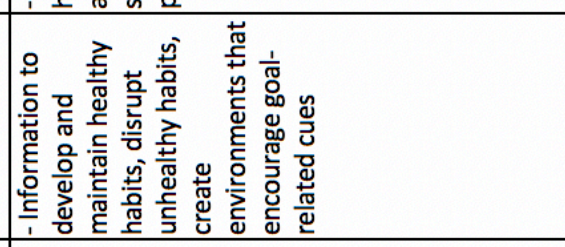 \\
\hline 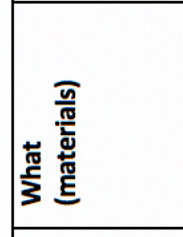 & 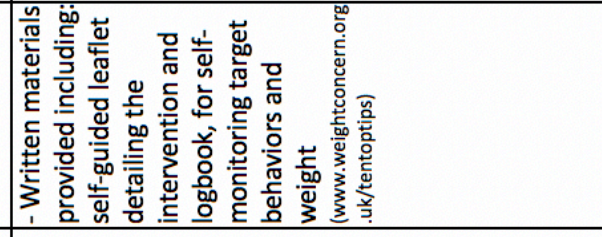 & 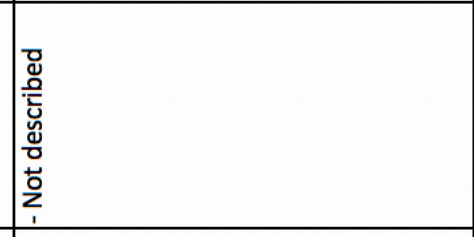 & \\
\hline$\vec{z}$ & 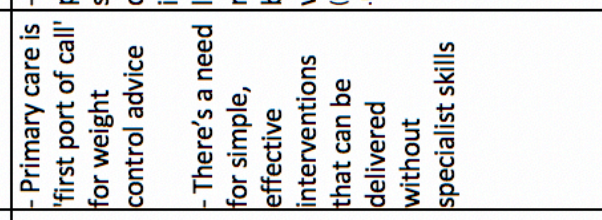 & 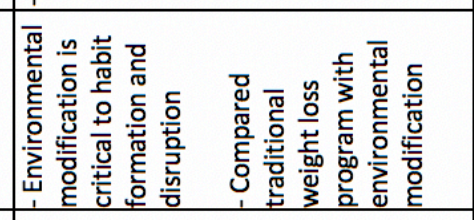 & 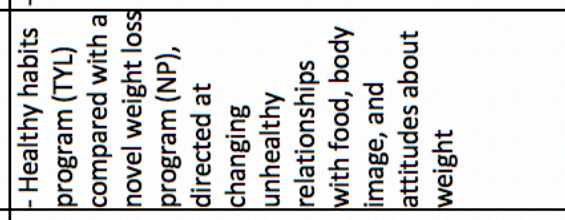 \\
\hline & 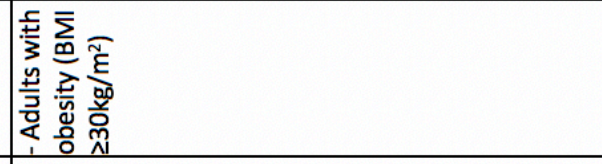 & 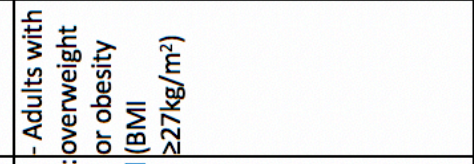 & 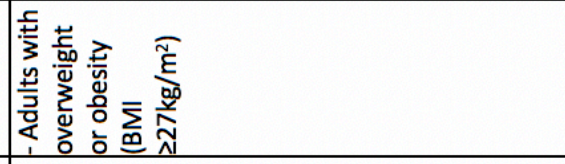 \\
\hline 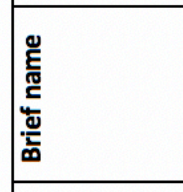 & 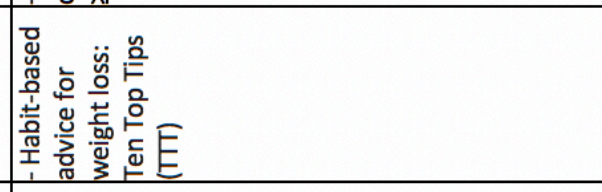 & 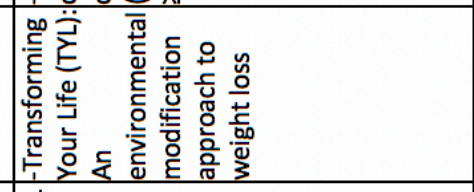 & 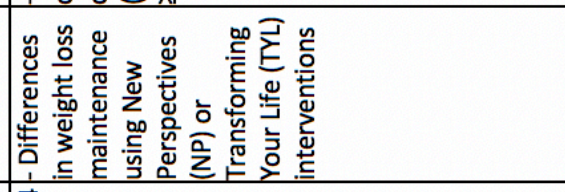 \\
\hline 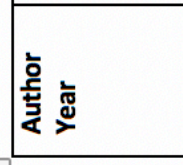 & 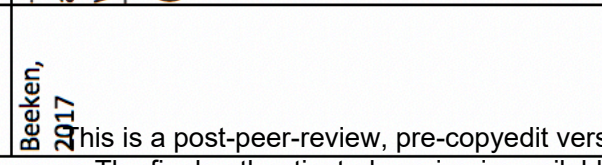 & 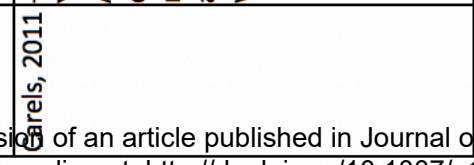 & 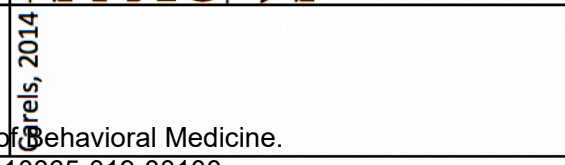 \\
\hline
\end{tabular}




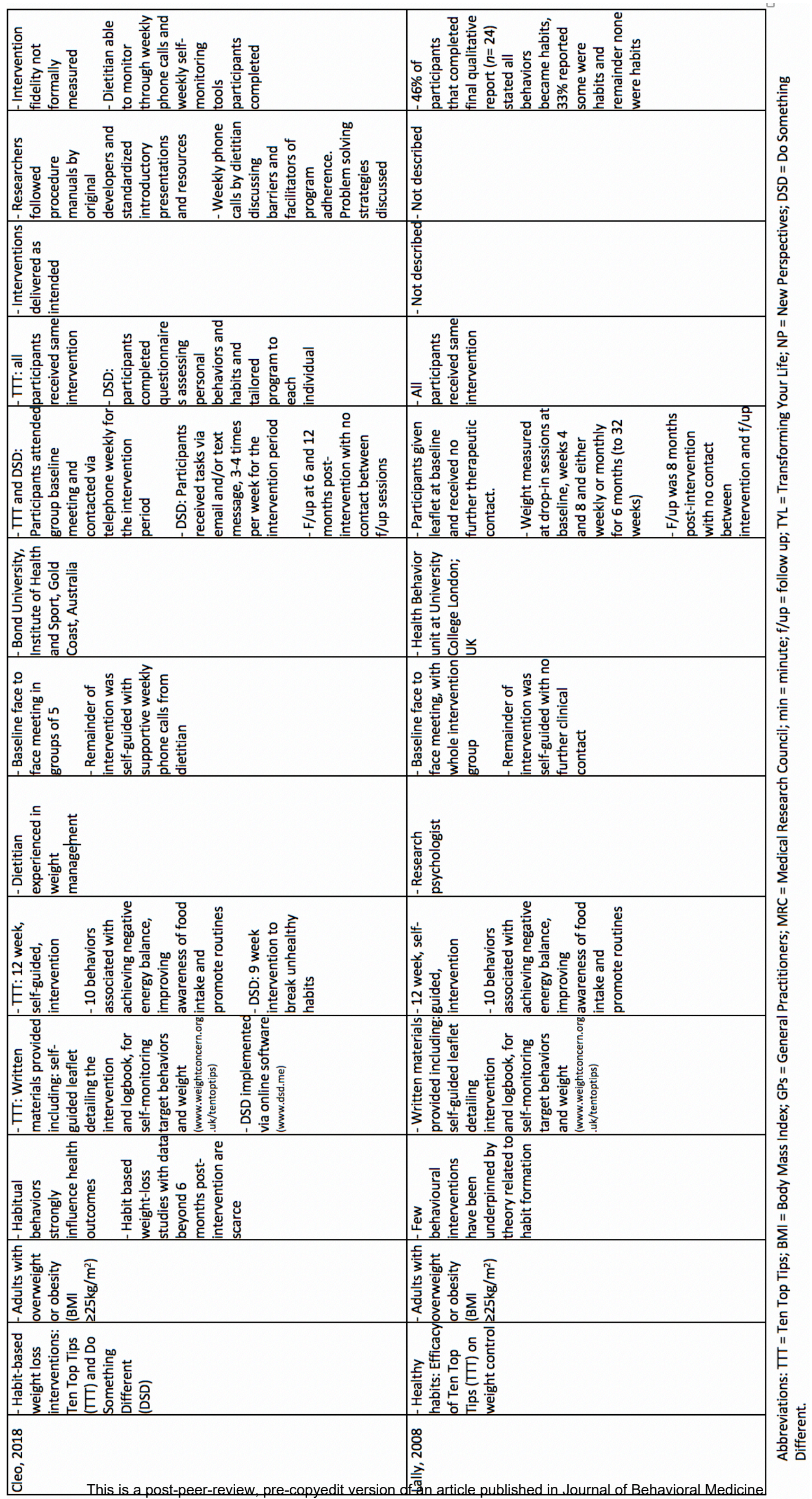


Cleo, 2019. Supplementary Material.

Title: Efficacy of habit-based weight loss interventions: a systematic review and metaanalysis

Authors: Gina Cleo, Elaine Beller, Paul Glasziou, Elisabeth Isenring, Rae Thomas

\section{Supplementary Material}

Supplementary File 1: PROSPERO International prospective register of systematic reviews

Supplementary File 2: Search Strategy

Supplementary Table 1: Descriptive data of excluded studies

Supplementary Figure 1: Comparing weight loss $(\mathrm{kg})$ at follow-up between intervention and control groups

Supplementary Figure 2: Proportion of participants achieving $\geq 5 \%$ total body weight reduction at post-intervention and follow-up

Supplementary Figure 3: Subgroup analysis comparing weight loss $(\mathrm{kg})$ between forming habits and a combination of forming and breaking habits 
Cleo, 2019. Supplementary Material.

\section{Supplementary File 1: PROSPERO International prospective register of systematic reviews}

UNIVERSITY oflfork

Centre for Reviews and Dissemination

\section{PROSPERO International prospective register of systematic reviews}

Review title and timescale

1 Review title

Give the working title of the review. This must be in English. Ideally it should state succinctly the interventions or exposures being reviewed and the associated health or social problem being addressed in the review.

Efficacy of habit-based weight loss interventions: a systematic review and meta analysis

2 Original language title

For reviews in languages other than English, this field should be used to enter the title in the language of the review. This will be displayed together with the English language title.

3 Anticipated or actual start date

Give the date when the systematic review commenced, or is expected to commence. 02/06/2017

4 Anticipated completion date

Give the date by which the review is expected to be completed. 08/09/2017

5 Stage of review at time of this submission

Indicate the stage of progress of the review by ticking the relevant boxes. Reviews that have progressed beyond the point of completing data extraction at the time of initial registration are not eligible for inclusion in PROSPERO. This field should be updated when any amendments are made to a published record.

The review has not yet started

$\times$

Review stage

Preliminary searches

Piloting of the study selection process

Formal screening of search results against eligibility criteria

Data extraction

Risk of bias (quality) assessment

Data analysis

$\begin{array}{cc}\text { Started } & \text { Completed } \\ \text { Yes } & \text { Yes } \\ \text { Yes } & \text { Yes } \\ \text { Yes } & \text { Yes } \\ \text { Yes } & \text { No } \\ \text { No } & \text { No } \\ \text { No } & \text { No }\end{array}$

Provide any other relevant information about the stage of the review here.

Review team details

$6 \quad$ Named contact

The named contact acts as the guarantor for the accuracy of the information presented in the register record.

Ms Cleo

$7 \quad$ Named contact email

Enter the electronic mail address of the named contact. gcleo@bond.edu.au

$8 \quad$ Named contact address

Enter the full postal address for the named contact.

Center for Research in Evidence Based Practice Faculty of Health Sciences and Medicine Bond University 14

University Drive, ROBINA QLD 4226 AUSTRALIA

$9 \quad$ Named contact phone number

Enter the telephone number for the named contact, including international dialing code.

+61755951588

10 Organisational affiliation of the review

Full title of the organisational affiliations for this review, and website address if available. This field may be completed as 'None' if the review is not affiliated to any organisation. 


\title{
UNIVERSITY of Work \\ Centre for Reviews and Dissemination
}

\author{
Bond University
}

Website address:

https://bond.edu.au/

11 Review team members and their organisational affiliations

Give the title, first name and last name of all members of the team working directly on the review. Give the organisational affiliations of each member of the review team.

$\begin{array}{llll}\text { Title } & \text { First name } & \text { Last name } & \text { Affiliation } \\ \text { Ms } & \text { Gina } & \text { Cleo } & \text { Bond University } \\ \text { Professor } & \text { Paul } & \text { Glasziou } & \text { Bond University } \\ \text { Professor } & \text { Elaine } & \text { Beller } & \text { Bond University } \\ \text { Dr } & \text { Rae } & \text { Thomas } & \text { Bond University }\end{array}$

\section{Funding sources/sponsors}

Give details of the individuals, organizations, groups or other legal entities who take responsibility for initiating, managing, sponsoring and/or financing the review. Any unique identification numbers assigned to the review by the individuals or bodies listed should be included.

This review received no funding sources or sponsors $G C$ received no funding with respect to this study. RT and EB are supported by a NHMRC grant (APP1104136, APP1044904). PG is supported by a NHMRC Australian Fellowship grant (GNT1080042).

13 Conflicts of interest

List any conditions that could lead to actual or perceived undue influence on judgements concerning the main topic investigated in the review.

Are there any actual or potential conflicts of interest?

Yes

$\mathrm{GC}$ has conducted a randomized controlled trial comparing two habit-based interventions for weight loss. Manuscript is under review.

\section{Collaborators}

Give the name, affiliation and role of any individuals or organisations who are working on the review but who are not listed as review team members.

$\begin{array}{llll}\text { Title } & \text { First name } & \text { Last name } & \text { Organisation details } \\ \mathrm{Ms} & \text { Rebecca } & \text { Sims } & \text { Bond University } \\ \mathrm{Mr} & \text { Justin } & \text { Clark } & \text { Bond University }\end{array}$

\section{Review methods}

15 Review question(s)

State the question(s) to be addressed / review objectives. Please complete a separate box for each question. What is the effect of habit-based weight-loss interventions on weight reduction in overweight and obese adults?

What is the effect of habit-based weight-loss interventions on weight loss maintenance in overweight and obese adults after the conclusion of the study (at last follow-up)?

What is the proportion of participants achieving clinically important weight loss and weight loss maintenance $(>/=5 \%$ total body weight loss) post-intervention and at last follow-up?

\section{Searches}

Give details of the sources to be searched, and any restrictions (e.g. language or publication period). The full search strategy is not required, but may be supplied as a link or attachment.

Electronic searches within PubMed, Embase, CINAHL, Cochrane CENTRAL and Web of Science, will be conducted to identify potential studies for inclusion. The search strategy will include only terms relating to or describing habitbased weight loss interventions. There will be no language or date restrictions. The searches will be re-run just before the final analyses and further studies retrieved for inclusion. 


\section{UNIVERSITY of York \\ Centre for Reviews and Dissemination}

\section{URL to search strategy}

If you have one, give the link to your search strategy here. Alternatively you can e-mail this to PROSPERO and we will store and link to it.

https://www.crd.york.ac.uk/PROSPEROFILES/65589_STRATEGY_20170619.pdf

I give permission for this file to be made publicly available

Yes

18 Condition or domain being studied

Give a short description of the disease, condition or healthcare domain being studied. This could include health and wellbeing outcomes.

Overweight and/or obesity

19 Participants/population

Give summary criteria for the participants or populations being studied by the review. The preferred format includes details of both inclusion and exclusion criteria.

Inclusion: Adults (18 years of age and above); overweight or obese by BMI classification $\left(>/=25.0 \mathrm{~kg} / \mathrm{m}^{\wedge} 2\right)$ Exclusion: Children or adolescents (under 18 years of age).

20 Intervention(s), exposure(s)

Give full and clear descriptions of the nature of the interventions or the exposures to be reviewed

The search strategy will restrict the search to habit-based weight-loss interventions that focus specifically on habitchange as the conceptual theory; therefore, habit-change is the primary strategy for behavior change and weight loss. The inclusion criteria of studies for review are: randomized clinical trials and quasi randomized clinical trials that quantify weight change outcomes in overweight or obese adults (at a minimum for pre-intervention and postintervention), any year, country or language. If multiple post-intervention follow-up assessments are reported, then the longest follow-up (i.e., longest duration from the conclusion of the intervention) will be used for the purpose of this review.

21 Comparator(s)/control

Where relevant, give details of the altematives against which the main subject/topic of the review will be compared (e.g. another intervention or a non-exposed control group).

The comparator is likely to be a waitlist comparison group, usual care group, or an alternative active intervention group.

22 Types of study to be included

Give details of the study designs to be included in the review. If there are no restrictions on the types of study design eligible for inclusion, this should be stated.

We will include randomized clinical trials and quasi randomised clinical trials which report weight change outcomes. The trial must include an intervention and a control group.

23 Context

Give summary details of the setting and other relevant characteristics which help define the inclusion or exclusion criteria.

Trials conducted in any setting and any country will be included.

24 Primary outcome(s)

Give the most important outcomes.

Change in weight $(\mathrm{kg})$

Give information on timing and effect measures, as appropriate.

Changes in weight from (1) baseline to post-intervention and (2) baseline to last available follow-up.

25 Secondary outcomes

List any additional outcomes that will be addressed. If there are no secondary outcomes enter None.

Clinically beneficial weight change (weight loss $=>/=5 \%$ total body weight)

Give information on timing and effect measures, as appropriate.

Proportion of participants losing $>/=5 \%$ total body weight lost at last available follow-up 


\section{UNIVERSITY of Work Centre for Reviews and Dissemination}

26 Data extraction (selection and coding)

Give the procedure for selecting studies for the review and extracting data, including the number of researchers involved and how discrepancies will be resolved. List the data to be extracted.

Titles and/or abstracts of studies retrieved using the search strategy and those from additional sources will be screened independently by two reviewers to identify studies that potentially meet the inclusion criteria outlined above. The full text of these potentially eligible studies will be retrieved and independently assessed for eligibility by two review team members. Any disagreements will be resolved through discussion with a third reviewer. A standardized, pre-piloted form will be used to extract data from the included studies for assessment of study quality and evidence synthesis. Extracted information will include: study setting; study population and participant demographics and baseline characteristics; details of the intervention and control conditions; type of study used; recruitment and study completion dates; outcomes and times of measurement; information for assessment of the risk of bias. Two reviewers will extract data independently, discrepancies will be identified and resolved through discussion, consensus, or arbiter. Missing data will be requested from study authors via email at least twice.

27 Risk of bias (quality) assessment

State whether and how risk of bias will be assessed, how the quality of individual studies will be assessed, and whether and how this will influence the planned synthesis.

Two independent reviewers will assess the risk of bias in included studies by using the Cochrane Risk of Bias tool (Higgins \& Green 2011). This includes: random sequence generation (selection bias); allocation concealment (selection bias); blinding of participants and personnel (performance bias); blinding of outcome assessment (detection bias); incomplete outcome data (attrition bias); selective reporting (reporting bias); other sources of bias. Each of these domains will be described as reported in the trial and then assigned a judgement about the adequacy of each item: 'low', 'high', or 'unclear' risk of bias. Disagreements between reviewers will be resolved by discussion, consensus, or arbiter.

28 Strategy for data synthesis

Give the planned general approach to be used, for example whether the data to be used will be aggregate or at the level of individual participants, and whether a quantitative or narrative (descriptive) synthesis is planned. Where appropriate a brief outline of analytic approach should be given.

We anticipate that there will be limited scope for meta-analysis due to heterogeneity of intervention duration and methods. However, where studies have used the same type of intervention and comparator, we will pool the results using a random-effects meta-analysis and calculate $95 \%$ confidence intervals and two sided $\mathrm{P}$ values for each outcome. We will also provide a narrative synthesis of the findings from the included studies, structured around the type of intervention, target population characteristics, type of outcome and intervention content. We will provide summaries of intervention effects for each study by calculating mean differences for changes in weight. We will describe the proportion of participants who have lost . $/=5 \%$ total body weight from baseline to post-intervention and baseline to last available follow-up.

29 Analysis of subgroups or subsets

Give any planned exploration of subgroups or subsets within the review. 'None planned' is a valid response if no subgroup analyses are planned.

Active vs non-active controls

\section{Review general information}

30 Type and method of review

Select the type of review and the review method from the drop down list.

Systematic review

31 Language

Select the language(s) in which the review is being written and will be made available, from the drop down list. Use the control key to select more than one language.

English

Will a summary/abstract be made available in English?

Yes

32 Country

Select the country in which the review is being carried out from the drop down list. For multi-national collaborations select all the countries involved. Use the control key to select more than one country.

Australia 


\section{UNIVERSITY of York \\ Centre for Reviews and Dissemination}

33 Other registration details

Give the name of any organisation where the systematic review title or protocol is registered together with any unique identification number assigned. If extracted data will be stored and made available through a repository such as the Systematic Review Data Repository (SRDR), details and a link should be included here.

34 Reference and/or URL for published protocol

Give the citation for the published protocol, if there is one.

Give the link to the published protocol, if there is one. This may be to an external site or to a protocol deposited with CRD in pdf format.

I give permission for this file to be made publicly available

Yes

35 Dissemination plans

Give brief details of plans for communicating essential messages from the review to the appropriate audiences.

Outcomes of this review and meta-analysis are expected to be published in a peer-reviewed journal.

Do you intend to publish the review on completion?

Yes

36 Keywords

Give words or phrases that best describe the review. (One word per box, create a new box for each term) weight management

behaviour change

habit-change

habit-based intervention

weight loss intervention

weight loss maintenance

systematic review

forming habits

breaking habits

37 Details of any existing review of the same topic by the same authors

Give details of earlier versions of the systematic review if an update of an existing review is being registered, including full bibliographic reference if possible.

38 Current review status

Review status should be updated when the review is completed and when it is published. Ongoing

39 Any additional information

Provide any further information the review team consider relevant to the registration of the review.

40 Details of final report/publication(s)

This field should be left empty until details of the completed review are available.

Give the full citation for the final report or publication of the systematic review.

Give the URL where available. 
Cleo, 2019. Supplementary Material.

\section{Supplementary File 2: Search Strategy}

We used the following search strategies to search Pubmed to search the Cochrane Central Register of Controlled Trials (CENTRAL), Embase, CINAHL and Web of Science. There were no language or date restrictions.

\section{PubMed search}

("Habits"[Mesh] OR “Habits"[tiab] OR Habit[tiab])

AND

(Formation[tiab] OR Disruption[tiab] OR Based[tiab] OR Breaking[tiab] OR Break[tiab])

AND

("Weight Loss"[Mesh] OR “Weight loss"[tiab] OR “Weight control”[tiab] OR “Weight reduction"[tiab])

AND

("Patient Education as Topic"[Mesh] OR "Pamphlets"[Mesh] OR "Diet, Reducing"[Mesh] OR "Weight Reduction Programs"[Mesh] OR "Environment Design"[Mesh] OR "Patient education"[tiab] OR Pamphlet[tiab] OR Pamphlets[tiab] OR Leaflet[tiab] OR Leaflets[tiab] OR Dietary[tiab] OR Diet[tiab] OR Diets[tiab] OR Food[tiab] OR Foods[tiab] OR "Weight Reduction Programs"[tiab] OR "Weight Reducing Programs"[tiab] OR "Weight Reduction Program"[tiab] OR "Weight Reducing Program"[tiab] OR "Environment Design"[tiab] OR “Environmental modification"[tiab])

AND

("randomized controlled trial"[pt] OR "controlled clinical trial" [pt] OR randomized[tiab] OR randomised[tiab] OR placebo[tiab] OR randomly[tiab] OR trial[tiab] OR groups[tiab] OR "Epidemiologic Studies"[Mesh] OR “case-control studies"[Mesh] OR “Cohort Studies"[Mesh] OR "case control"[tiab] OR Cohort[tiab] OR "Follow up"[tiab] OR Observational[tiab] OR longitudinal[tiab] OR Prospective[tiab] OR retrospective[tiab] OR "cross sectional" [tiab] OR "CrossSectional Studies"[Mesh] OR Investigated[tiab] OR Analysis[tiab] OR Statistics[tiab] OR Data[tiab] OR "statistics and numerical data"[sh] OR "epidemiology"[sh]) 
Cleo, 2019. Supplementary Material.

\section{Cochrane CENTRAL}

([mh Habits] OR Habits:ti,ab OR Habit:ti,ab)

AND

(Formation:ti,ab OR Disruption:ti,ab OR Based:ti,ab OR Breaking:ti,ab OR Break:ti,ab)

AND

([mh "Weight Loss"] OR "Weight loss":ti,ab OR "Weight control":ti,ab OR "Weight reduction":ti,ab)

AND

([mh "Patient Education as Topic"] OR [mh Pamphlets] OR [mh "Diet, Reducing"] OR [mh "Weight Reduction Programs"] OR [mh "Environment Design"] OR "Patient education":ti,ab OR Pamphlet:ti,ab OR Pamphlets:ti,ab OR Leaflet:ti,ab OR Leaflets:ti,ab OR Dietary:ti,ab OR Diet:ti,ab OR Diets:ti,ab OR Food:ti,ab OR Foods:ti,ab OR "Weight Reduction Programs":ti,ab OR "Weight Reducing Programs":ti,ab OR "Weight Reduction Program":ti,ab OR "Weight Reducing Program":ti,ab OR "Environment Design":ti,ab OR "Environmental modification":ti,ab)

\section{Embase}

('Habit'/exp OR Habits:ti,ab OR Habit:ti,ab)

AND

(Formation:ti,ab OR Disruption:ti,ab OR Based:ti,ab OR Breaking:ti,ab OR Break:ti,ab)

AND

('weight reduction'/exp OR "Weight loss":ti,ab OR "Weight control":ti,ab OR "Weight reduction":ti,ab)

AND

('Patient Education'/exp OR 'publication'/exp OR 'diet therapy'/exp OR 'weight loss program'/exp OR 'environmental planning'/exp OR "Patient education":ti,ab OR Pamphlet:ti,ab OR Pamphlets:ti,ab OR Leaflet:ti,ab OR Leaflets:ti,ab OR Dietary:ti,ab OR Diet:ti,ab OR Diets:ti,ab OR Food:ti,ab OR Foods:ti,ab OR "Weight Reduction Programs":ti,ab OR "Weight Reducing Programs":ti,ab OR "Weight Reduction Program":ti,ab OR "Weight Reducing Program":ti,ab OR "Environment Design":ti,ab OR "Environmental modification":ti,ab)

AND

(random* OR factorial OR crossover OR placebo OR blind OR blinded OR assign OR assigned OR allocate OR allocated OR 'crossover procedure'/exp OR 'double-blind procedure'/exp OR 'randomized controlled trial'/exp OR 'single-blind procedure'/exp OR 'epidemiology'/exp OR 'controlled study'/exp OR 'cohort analysis'/exp OR "case control":ti,ab OR Cohort:ti,ab OR "Follow 
Cleo, 2019. Supplementary Material.

up":ti,ab OR Observational:ti,ab OR longitudinal:ti,ab OR Prospective:ti,ab OR retrospective:ti,ab OR "cross sectional":ti,ab OR 'Cross-Sectional Studies'/exp OR Investigated:ti,ab OR Analysis:ti,ab OR Statistics:ti,ab OR Data:ti,ab)

\section{CINAHL}

((MH "Habits+") OR TI Habits OR AB Habits OR TI Habit OR AB Habit)

AND

(TI Formation OR AB Formation OR TI Disruption OR AB Disruption OR TI Based OR AB Based OR TI Breaking OR AB Breaking OR TI Break OR AB Break)

AND

((MH "Weight Loss+") OR TI "Weight loss" OR AB "Weight loss" OR TI "Weight control" OR AB "Weight control" OR TI "Weight reduction" OR AB "Weight reduction")

AND

((MH "Patient Education+") OR (MH "Pamphlets+") OR (MH "Diet, Reducing+") OR (MH "Weight Reduction Programs+") OR TI "Patient education" OR AB "Patient education" OR TI Pamphlet OR AB Pamphlet OR TI Pamphlets OR AB Pamphlets OR TI Leaflet OR AB Leaflet OR TI Leaflets OR AB Leaflets OR TI Dietary OR AB Dietary OR TI Diet OR AB Diet OR TI Diets OR AB Diets OR TI Food OR AB Food OR TI Foods OR AB Foods OR TI "Weight Reduction Programs" OR AB "Weight Reduction Programs" OR TI "Weight Reducing Programs" OR AB "Weight Reducing Programs" OR TI "Weight Reduction Program" OR AB "Weight Reduction Program" OR TI "Weight Reducing Program" OR AB "Weight Reducing Program" OR TI "Environment Design" OR AB "Environment Design" OR TI "Environmental modification" OR AB "Environmental modification")

AND

((MH "Clinical Trials+") OR (MH "Quantitative Studies") OR TI placebo* OR AB placebo* OR (MH "Placebos") OR (MH "Random Assignment") OR TI random* OR AB random* OR TI ((singl* or doubl* or tripl* or trebl*) W1 (blind* or mask*)) OR AB ((singl* or doubl* or tripl* or trebl*) W1 (blind* or mask*)) OR TI clinic* trial* OR AB clinic* trial* OR PT clinical trial OR (MH "Epidemiological Research+") OR (MH "Study Design+") OR TI "case control" OR AB "case control" OR TI Cohort OR AB Cohort OR TI "Follow up" OR AB "Follow up" OR TI Observational OR AB Observational OR TI longitudinal $O R A B$ longitudinal $O R$ TI Prospective $O R A B$ Prospective $O R$ TI retrospective $O R A B$ retrospective $O R T I$ "cross sectional" OR AB "cross sectional" OR TI Investigated OR AB Investigated OR TI Analysis OR AB Analysis OR TI Statistics OR AB Statistics OR TI Data OR AB Data) 
Cleo, 2019. Supplementary Material.

\section{Web of Science}

(Habits OR Habit)

AND

(Formation OR Disruption OR Breaking OR Break)

AND

("Weight Loss" OR "Weight loss" OR "Weight control" OR "Weight reduction")

AND

("Patient Education as Topic" OR Pamphlets OR "Diet, Reducing" OR "Weight Reduction Programs" OR "Environment Design" OR "Patient education" OR Pamphlet OR Pamphlets OR Leaflet OR Leaflets OR Dietary OR Diet OR Diets OR "Weight Reduction Programs" OR "Weight Reducing Programs" OR "Weight Reduction Program" OR "Weight Reducing Program" OR "Environment Design" OR "Environmental modification")

AND

(Trial OR randomized OR randomised OR placebo OR randomly OR groups OR "Epidemiologic Studies" OR Epidemiological OR "case-control studies" OR "Cohort Studies" OR "case control" OR Cohort OR "Follow up" OR Observational OR longitudinal OR Prospective OR retrospective OR "cross sectional" OR "Cross-Sectional Studies" OR Investigated OR Analysis OR Statistics OR Data) 
Cleo, 2019. Supplementary Material.

Supplementary Table 1 Descriptive data of excluded studies

\begin{tabular}{|c|c|c|c|c|c|c|}
\hline Study reference & $\begin{array}{l}\text { Baseline weight, } \\
\text { kg* }\end{array}$ & Mean age, years & $\begin{array}{l}\text { Intervention } \\
\text { theory }\end{array}$ & $\begin{array}{l}\text { Duration of } \\
\text { intervention } \\
\text { (weeks) }\end{array}$ & $\begin{array}{l}\text { Follow-up } \\
\text { (months) }\end{array}$ & $\begin{array}{l}\text { Reason for } \\
\text { exclusion }\end{array}$ \\
\hline Beeken, 2011 & $\begin{array}{l}\text { Pre-intervention } \\
\text { (no data) }\end{array}$ & $\begin{array}{l}\text { Pre-intervention } \\
\text { (no data) }\end{array}$ & Forming habits & 12 & 24 & Duplicate \\
\hline Beeken, 2014 & $100.8( \pm 17.2)$ & 59 (IQR 48.7-66.8) & Forming habits & 12 & 24 & Duplicate \\
\hline Fletcher, 2011 & $29.6 \mathrm{~kg} / \mathrm{m}^{2}( \pm 4.8)$ & $44.2( \pm 7.3)$ & Breaking habits & 4 & 2 & Non-randomised \\
\hline Kraschnewski, 2010 & $93.2( \pm 14.4)$ & $50( \pm 10.9)$ & Lifestyle program & 12 & nil & Not habit-based \\
\hline Page, 2008 & $30 \mathrm{~kg} / \mathrm{m}^{2}( \pm 5.7)$ & $44.4( \pm 8.7)$ & Breaking habits & 4 & $2-3$ & Non-randomised \\
\hline Ter Bogt, 2011 & 88 & $56( \pm 7.7)$ & Lifestyle program & $1 \times$ baseline session & $\begin{array}{l}8 \text { (visits) } \\
12 \text { (telephone) }\end{array}$ & Not habit-based \\
\hline
\end{tabular}

Abbreviations: $\mathrm{kg}=$ kilogram; $\mathrm{BMI}=$ Body Mass Index; $\mathrm{QQR}=$ Interquartile range.

* $\mathrm{BMI}\left(\mathrm{kg} / \mathrm{m}^{2}\right)$ was used if weight $(\mathrm{kg})$ not reported 
Cleo, 2019. Supplementary Material.

\begin{tabular}{|c|c|c|c|c|c|c|c|c|c|}
\hline \multirow[b]{2}{*}{ Study or Subgroup } & \multicolumn{3}{|c|}{ Intervention } & \multicolumn{3}{|c|}{ Control } & \multicolumn{2}{|r|}{ Mean Difference } & \multirow{2}{*}{$\begin{array}{l}\text { Mean Difference } \\
\text { IV, Random, } 95 \% \mathrm{Cl}\end{array}$} \\
\hline & Mean & SD & Total & Mean & SD & Total & Weight & IV, Random, $95 \% \mathrm{Cl}$ & \\
\hline Beeken, 2017 & -2.15 & 5.75 & 155 & -2.96 & 7.16 & 158 & $53.7 \%$ & $0.81[-0.63,2.25]$ & \\
\hline Carels, 2011 & -3.54 & 5.44 & 21 & -4.22 & 4.4 & 21 & $32.9 \%$ & $0.68[-2.31,3.67]$ & \\
\hline Carels, 2014 & -8.89 & 11.97 & 20 & -3.49 & 5.4 & 15 & $13.4 \%$ & $-5.40[-11.32,0.52]$ & \\
\hline Total $(95 \% \mathrm{Cl})$ & & & 196 & & & 194 & $100.0 \%$ & $-0.06[-2.48,2.36]$ & \\
\hline $\begin{array}{l}\text { Heterogeneity: Tau } \\
\text { Test for overall effec }\end{array}$ & $\begin{array}{l}2.30 ; C \\
Z=0.05\end{array}$ & $\begin{array}{l}1 i^{2}=4.0 \\
(P=0 .\end{array}$ & $\begin{array}{l}1, \mathrm{df}= \\
96)\end{array}$ & $2(\mathrm{P}=$ & $(13) ; i^{2}$ & $=50 \%$ & & & 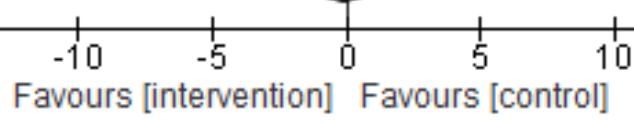 \\
\hline
\end{tabular}

Supplementary Figure 1 Comparing weight loss $(\mathrm{kg})$ at follow-up between intervention and control groups 


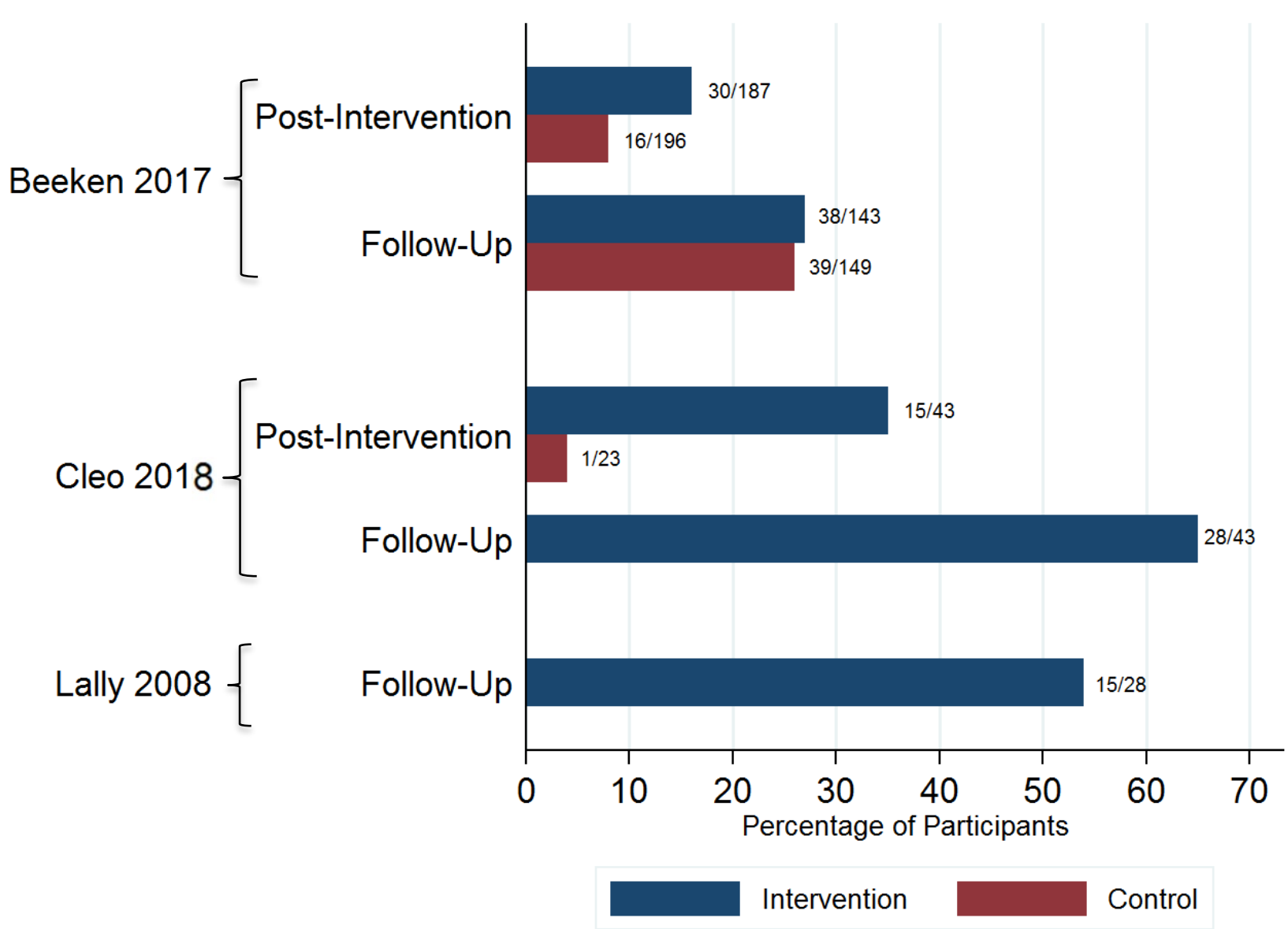

Supplementary Figure 2 Proportion of participants achieving $\geq 5 \%$ total body weight reduction at post-intervention and follow-up 


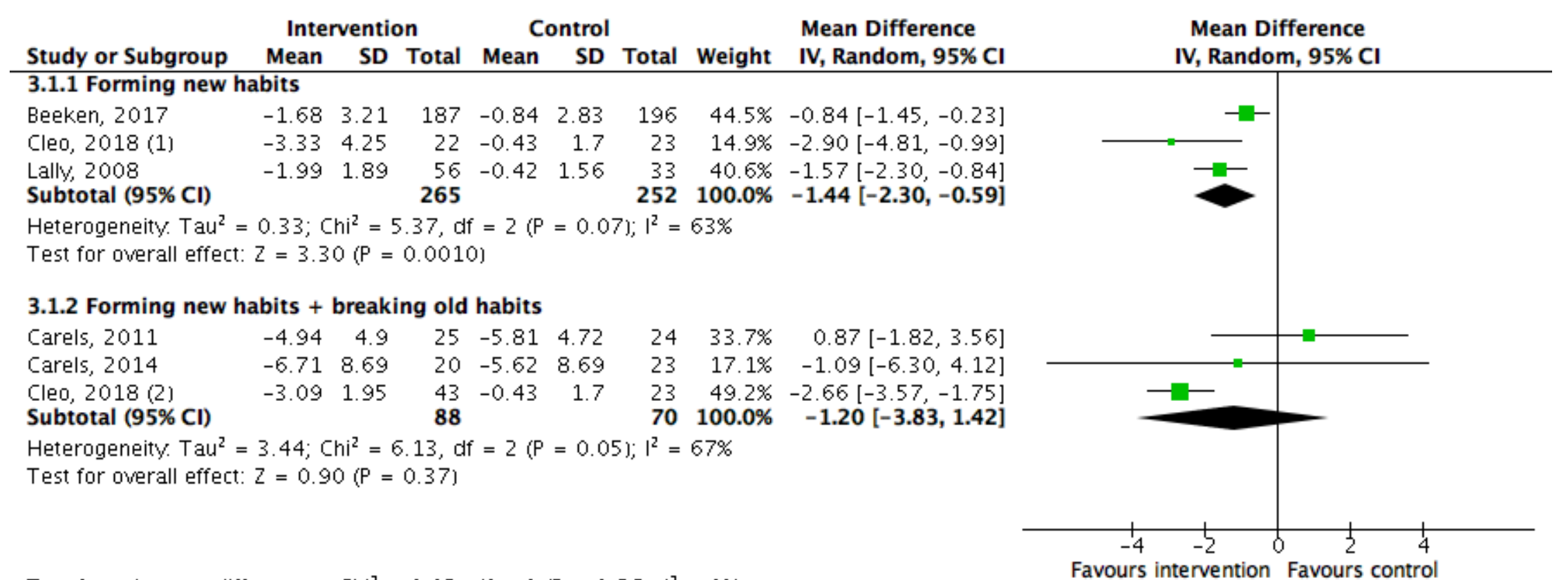

Test for subgroup differences: $C h i^{2}=0.03, d f=1(P=0.86), \mathrm{I}^{2}=0 \%$

Favours intervention Favours control Footnotes

(1) Data relate only to the habit forming intervention, Ten Top Tips, as reported in the publication.

(2) Data relate to the combined outcomes of the habit forming (Ten Top Tips) and habit breaking (Do Something Different) interventions.

Supplementary Figure 3 Subgroup analysis comparing weight loss $(\mathrm{kg})$ between forming habits and a combination of forming and breaking habits 